ARTICLE

https://doi.org/10.1038/s41467-019-08812-y

\title{
The human gut Firmicute Roseburia intestinalis is a primary degrader of dietary $\beta$-mannans
}

Sabina Leanti La Rosa (10 1, Maria Louise Leth², Leszek Michalak, Morten Ejby Hansen (1) 2, Nicholas A. Pudlo², Robert Glowacki ${ }^{3}$, Gabriel Pereira ${ }^{3}$, Christopher T. Workman², Magnus Ø. Arntzen ${ }^{1}$, Phillip B. Pope (1) 1, Eric C. Martens ${ }^{3}$, Maher Abou Hachem (i) ${ }^{2}$ \& Bjørge Westereng ${ }^{1}$

$\beta$-Mannans are plant cell wall polysaccharides that are commonly found in human diets. However, a mechanistic understanding into the key populations that degrade this glycan is absent, especially for the dominant Firmicutes phylum. Here, we show that the prominent butyrate-producing Firmicute Roseburia intestinalis expresses two loci conferring metabolism of $\beta$-mannans. We combine multi-"omic" analyses and detailed biochemical studies to comprehensively characterize loci-encoded proteins that are involved in $\beta$-mannan capturing, importation, de-branching and degradation into monosaccharides. In mixed cultures, $R$. intestinalis shares the available $\beta$-mannan with Bacteroides ovatus, demonstrating that the apparatus allows coexistence in a competitive environment. In murine experiments, $\beta$ mannan selectively promotes beneficial gut bacteria, exemplified by increased $R$. intestinalis, and reduction of mucus-degraders. Our findings highlight that $R$. intestinalis is a primary degrader of this dietary fiber and that this metabolic capacity could be exploited to selectively promote key members of the healthy microbiota using $\beta$-mannan-based therapeutic interventions.

\footnotetext{
${ }^{1}$ Faculty of Chemistry, Biotechnology and Food Science, Norwegian University of Life Sciences, Aas N-1433 Norge, Norway. ${ }^{2}$ Dept. of Biotechnology and Biomedicine, Danish Technical University, Kgs. Lyngby DK-2800, Denmark. ${ }^{3}$ Department of Microbiology and Immunology, University of Michigan Medical School, Ann Arbor 48109 MI, USA. Correspondence and requests for materials should be addressed to B.W. (email: bjorge.westereng@nmbu.no)
} 
T he human gastrointestinal tract harbors an extremely dense and diverse microbial community, known as the gut microbiota ${ }^{1}$. In a mutually beneficial relationship, the gut microbiota supplies enzymes able to depolymerize dietary carbohydrates that cannot be hydrolyzed by human enzymes ${ }^{2,3}$. The monosaccharides generated are further fermented into hostabsorbable metabolites, including the short-chain fatty acids butyrate, acetate, and propionate. In particular, butyrate produced by commensal bacteria serves as the main energy source for colonocytes ${ }^{4,5}$ and it exhibits anti-carcinogenic, anti-inflammatory, and barrier protective properties in the distal gut ${ }^{6-8}$. The relevance of this metabolic output to human health has prompted increasing interest in intentionally modulating the composition of the gut microbiota to promote wellbeing and combat disease, e.g., by the use of prebiotics ${ }^{9}$. Established prebiotics have been traditionally developed based on their selective fermentation by bifidobacteria and lactobacilli generally regarded as conferring health benefits to the host. Notably, other potentially beneficial targets are the butyrate-producing Firmicutes ${ }^{9}$.

Roseburia spp., together with Faecalibacterium prausnitzii and Eubacterium rectale, constitute a group of dominant butyrateproducing Firmicutes, estimated to account for $7-24 \%$ of the total bacteria in the healthy human colon ${ }^{10,11}$. Interest in Roseburia spp. has increased with reports that the abundance of these bacteria is reduced in individuals affected by inflammatory diseases $^{12-14}$ and colorectal cancer ${ }^{15,16}$. Complementary studies have shown that Roseburia spp. play an important role in the control of gut inflammatory processes ${ }^{17}$, amelioration of atherosclerosis $^{18}$ and in the maturation of the immune system, primarily through the production of butyrate ${ }^{19}$. R. intestinalis preferentially colonizes the mucin layer ${ }^{20,21}$ and this intimate association to the host may contribute to the local level of butyrate available for the colonic epithelial cells ${ }^{22}$. This species appears to be a specialist able to grow only on a few glycans ${ }^{23,24}$ and has been recently shown to be a prominent xylan degrader in vitro ${ }^{25}$ and in the healthy human colon $^{26}$.

$\beta$-Mannans are widespread in the human diet: they are widely used in food as thickening, stabilizing, and gelling agents ${ }^{27}$ (glucomannan and galactomannan, Fig. 1). They are found in the endospermic tissue of nuts (homopolymeric mannan), coffee beans, coconut palm, tomato, and legume seeds (galactomannan)

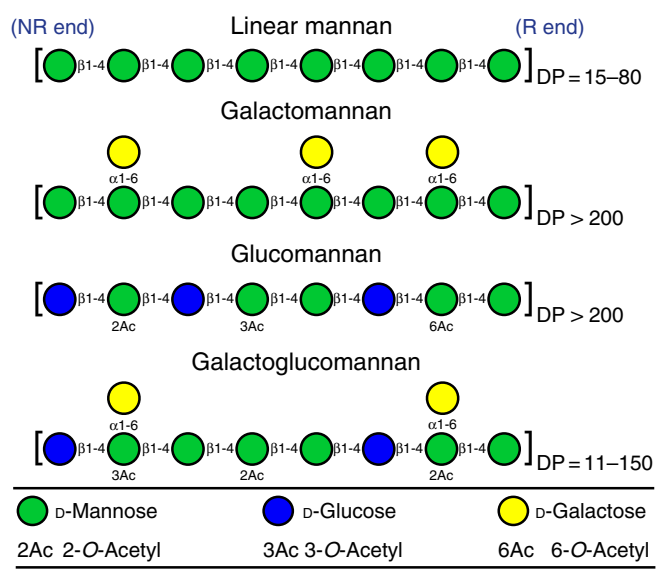

Fig. 1 General structure of the main classes of $\beta$-mannan. Linear homopolymeric (upper structure) and linear heteropolymeric (lower three structures) $\beta$-mannans are shown. Monosaccharides (D-mannose, green circle; D-glucose, blue circle; D-galactose, yellow circle) and acetylations (2Ac, 2-O-Acetyl; 3Ac, 3-O-Acetyl; 6Ac, 6-O-Acetyl) are represented using the standard Consortium of Functional Glycomics symbols ${ }^{67}$. NR end, nonreducing end; $R$ end, reducing end; $D P$, degree of polymerization
(Fig. 1 $)^{27-29}$, and play vital roles in the cell wall structure and as storage polysaccharides in plants. Notably, the structure of galactoglucomannan ${ }^{29}$ from non-edible sources (softwood) shares striking similarities with that from edible sources (Fig. 1).

Prevalent Gram-negative Bacteroides spp. encode $\beta$-mannan polysaccharide utilization loci (PULs) and have been recently shown to utilize mannans ${ }^{30-32}$. Despite members of the Firmicutes phylum being numerically dominant in the gut, insight is lacking into the metabolic strategies adopted by these Grampositive bacteria to utilize $\beta$-mannans.

Here, using a combination of microbiology,"omic" and enzymology approaches, we unravel the molecular mechanism evolved by $R$. intestinalis L1-82 to depolymerize $\beta$-mannans that are potentially available in the large intestine. Our findings show that $R$. intestinalis growth on $\beta$-mannan is dependent on the expression of a highly specific multi-modular cell attached endomannanase, an ATP-binding transporter and an intracellular enzyme cocktail through which linear and substituted manno-oligosaccharides are completely hydrolyzed to component monosaccharides for further metabolism. Using germ-free mice colonized with a model gut microbiota, we demonstrate that $\beta$-mannan alters the community composition, facilitating bacteria that have mannan degrading machineries. Besides extending the knowledge on the enzymatic basis of $\beta$-mannanmetabolism by members of the most numerous Firmicutes phylum, our results have implications for the design of targeted intervention strategies to manipulate the gut microbiota via supplementation of prebiotics to the diet to restore or improve health.

\section{Results}

Two multi-gene loci mediate $\beta$-mannan utilization. $R$. intestinalis L1-82 grows efficiently on a variety of complex $\beta$-mannans as a sole carbon source (Fig. 2a), causing a concomitant acidification of the medium (Fig. 2b). To evaluate which fractions of $\beta$-mannan breakdown products are internalized, we analyzed the culture supernatants during $R$. intestinalis growth on AcGGM using high-performance anion-exchange chromatography with pulsed amperometric detection (HPAEC-PAD) (Supplementary Fig. 1a, b). Neither oligosaccharides nor monosaccharides accumulated in the stationary phase culture (Supplementary Fig. 1a, b), indicating that the bacterium possesses a highly efficient apparatus to cleave and import all the sugars derived from the breakdown of this complex glycan.

To examine the molecular basis underlying $\beta$-mannan utilization by $R$. intestinalis, we performed an RNA sequencing (RNAseq) transcriptional analysis during growth on konjac glucomannan (KGM), spruce acetylated galactoglucomannan (AcGGM) and glucose (Glc). The top 20 upregulated genes in $\beta$-mannan transcriptome encode a $\beta$-mannanase belonging to the glycoside hydrolase (GH) 26 family (GH26 according to the CAZy classification ${ }^{33}$ ), a solute binding protein $(\mathrm{MnBP})$ and two permeases (MPP) of an $\mathrm{ABC}$ transporter, two phosphorylases (GH130), one epimerase (Mep), two $\beta$-glucosidases (GH3) and two carbohydrate esterases (CEs) (Fig. 2c and Supplementary Data 1). These genes are located in two loci, which were designated mannan-utilization locus large (MULL: ROSINTL182_05469-83) and mannan-utilization locus small (MULS: ROSINTL182_07683-85) (Fig. 2d). Among the MULL genes expression of a LacI-type transcriptional regulator, predicted glycosyl hydrolases belonging to GH113, GH36, GH1, and a phosphomutase also increased. The response was specific to $\beta$-mannan as no differential expression of these genes was observed during growth of $R$. intestinalis on galactose, a building block in mannan (Supplementary Table 1). 

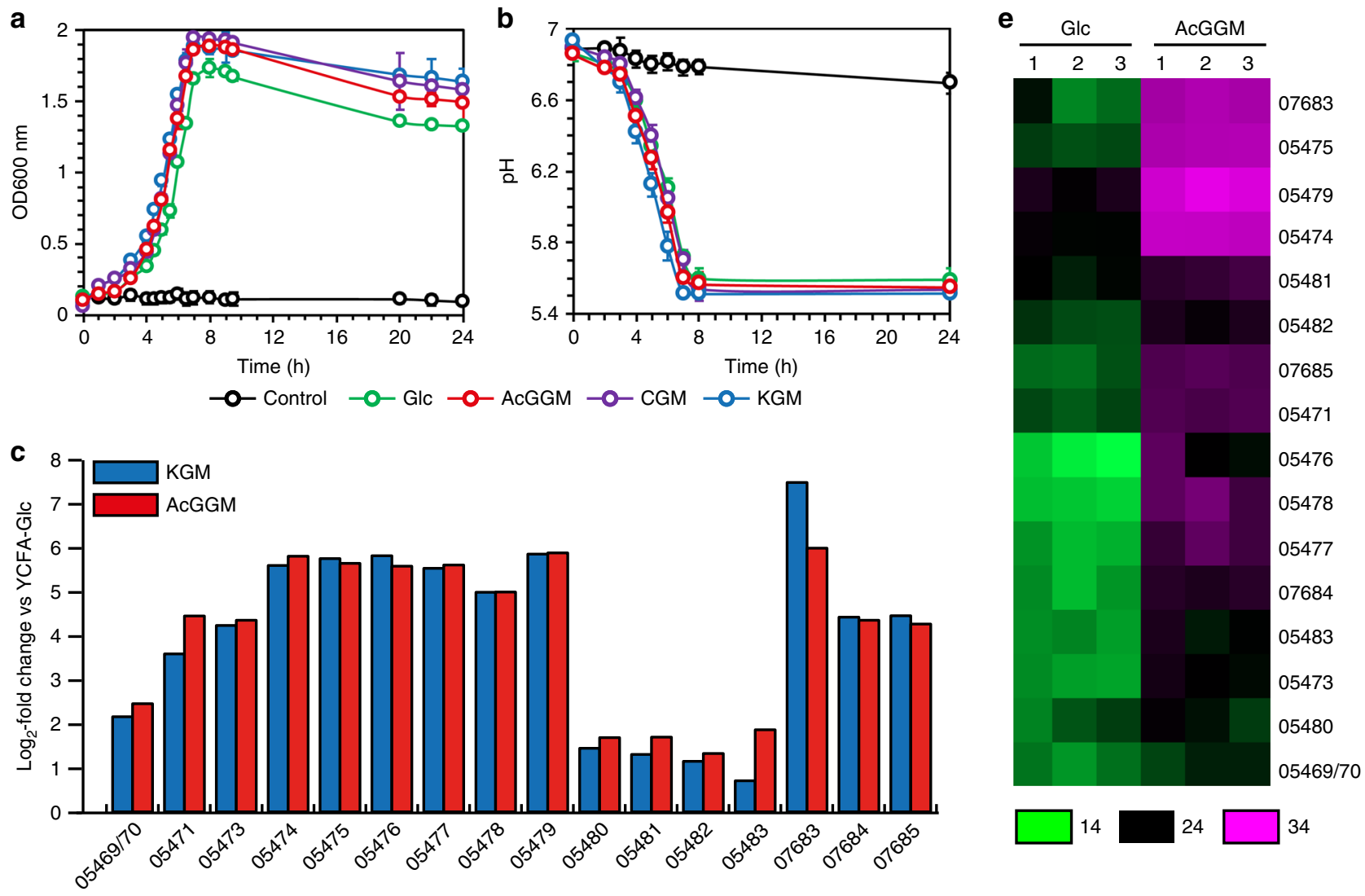

d MULL

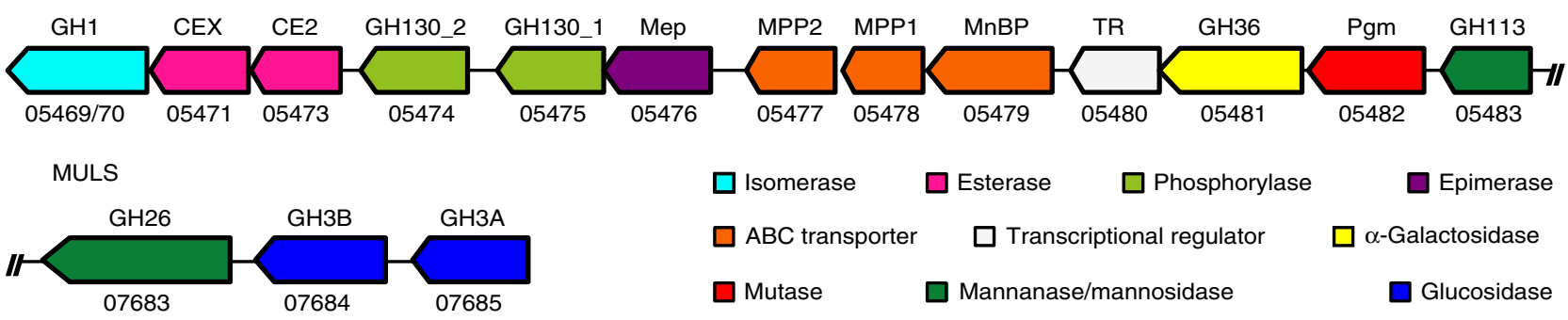

Fig. $2 R$. intestinalis upregulates several glycoside hydrolases, two carbohydrate esterases and an ABC-transporter during $\beta$-mannan consumption. a Growth curves of $R$. intestinalis in YCFA without carbon source (black) or supplemented with $0.5 \%$ of either glucose (Glc, green circles), KGM (blue circles), CGM (purple circles) or AcGGM (red circles). b pH measurements during $R$. intestinalis growth on Glc and $\beta$-mannans. In $\mathbf{a}$ and $\mathbf{b}$, each point on the curves represent the average of three independent experiments. Error bars represent standard deviations (s.d.). c RNA expression profile of putative $\beta$-mannan utilization genes during $R$. intestinalis L1-82 growth in YCFA supplemented with $0.5 \%$ KGM (blue bars) or AcGGM (red bars). The Log 2 -fold change relative to cells cultured on YCFA-GIc is shown on the $y$-axis while the $x$-axis shows the putative genes involved in $\beta$-mannan catabolism. $\mathbf{d}$ Genomic organization of the large and small $\beta$-mannan utilization loci (MULL and MULS, respectively) from $R$. intestinalis. Genes with similar predicted functions are coded by the same color. e Heat map showing the proteomic detection of relevant proteins with predicted $\beta$-mannan utilization functions in triplicate samples (1-3) grown on YCFA-GIc and YCFA-AcGGM. Colors represent protein intensity expressed as $\mathrm{Log}_{2}$ of LFQ values; the color gradient ranges from 14 (green) to 34 (magenta), with black indicating 24. In c-e, locus tag numbers ROSINTL182_XXXXX are abbreviated with the last numbers after the hyphen

Proteomic analysis under the same growth conditions corroborated the RNAseq results; indeed, proteins encoded by the genes located in MULL and MULS were abundant in the AcGGM samples compared to the glucose samples (Fig. 2e, Supplementary Data 2).

We carried out a comparative genomic analysis to establish the distribution of $\beta$-mannans utilization loci equivalent to the identified MULL and MULS in other representative Roseburia spp. and Clostridium cluster XIVa members. The results showed that $R$. faecis and $R$. hominis shared an overall MULL and MULS organization with that of $R$. intestinalis (Supplementary Fig. 2, Supplementary Table 2), suggesting that the utilization of $\beta$ mannan is shared by these three Roseburia spp. However, the lack of the critical GH26 endomannanase, required to break down mannan (see later results for $R$. intestinalis $\beta$-mannanase $R i \mathrm{GH} 26$ ), is likely to render $R$. hominis only able to metabolize manno-oligosaccharides. Orthologous mannan utilization loci were identified in specific members of the Clostridium cluster XIVa, although a similar organization and complete conservation of all MULL and MULS components was not observed (Supplementary Fig. 2).

Degradation of the $\boldsymbol{\beta}$-mannan backbone. RiGH26, (locus tag: ROSINTL182_07683), is a predicted extracellular modular $\beta$ mannanase comprising a carbohydrate binding module of family 


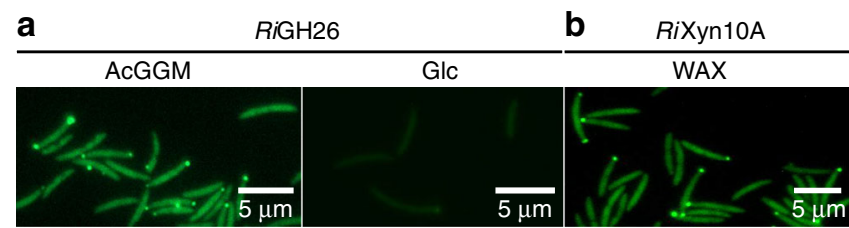

Fig. 3 Cellular location of the endomannanase RiGH26. a Fluorescent microscopy images of $R$. intestinalis cells cultured on AcGGM or Glc and incubated with polyclonal antibodies raised against the recombinant endomannanase RiGH26. Glucose-grown cells exhibit a low intensity fluorescence signal; this is consistent with the results of the proteomics data showing that, when the organism is cultured on glucose, $R i G H 26$ is expressed at basal levels. $\mathbf{b}$ Fluorescent microscopy images of $R$. intestinalis cells grown on WAX (positive control) and incubated with antibodies raised against the known surface endoxylanase RiXyn10A 25 . Localization microscopy images are representative data from two biological duplicates

27 (CBM27), a catalytic module of GH26 followed by a CBM23 (Supplementary Fig. 3a). Furthermore, two C-terminal Ig-like domains and a proline-glycine rich region likely mediate cell attachment $^{34}$ and binding within the cell wall ${ }^{35}$. The extracellular localization of $\mathrm{RiGH} 26$ was corroborated experimentally by immunofluorescence microscopy (Fig. 3). RiGH26 exhibited activity toward decorated mannans including KGM, carob galactomannan (CGM) and AcGGM (Fig. 4a and Supplementary Fig. 3b), generating linear and substituted mannooligosaccharides. The enzyme was active on mannopentaose $\left(\mathrm{M}_{5}\right)$ and mannotetraose $\left(\mathrm{M}_{4}\right)$ but not mannobiose $\left(\mathrm{M}_{2}\right)$ (Supplementary Fig. 3c). Overall, the product profiles suggest capability of endo-action and indicates that $\mathrm{RiGH} 26$ targets large polymers and can accommodate the galactose and acetyl decorations present in these substrates. Further analysis indicated that RiGH26 is a potent enzyme as, when used at the concentration of $10 \mathrm{nM}$, it was able to hydrolyze high concentrations of spruce AcGGM (50 mg ml-1$)$ into oligosaccharides in $1 \mathrm{~h}$ at standard assay conditions (Supplementary Fig. 3d). No detectable activity was measured when RiGH26 was incubated with linear cello-oligosaccharides, birch xylan, curdlan, lichenan or barley derived $\beta$-glucan, thus confirming the specificity of RiGH26 towards $\beta$-mannan (Supplementary Fig. 3c).

BlastP searches showed that homologs of RiGH26, including the two predicted carbohydrate binding modules CBM27 and CBM23, were exclusively found in $\beta$-mannanase encoded by Firmicutes belonging to various other members of the Clostridium cluster XIVa (Supplementary Fig. 4, Supplementary Table 3-5). To investigate the biochemical properties of the two modules, RiCBM27 and RiCBM23 were expressed in Escherichia coli and their capacities to bind to a range of different soluble cello-oligosaccharides and manno-oligosaccharides were evaluated using surface plasmon resonance (SPR). Recombinant RiCBM27 and RiCBM23 bound only manno-oligosaccharides (Table 1), but differed in their binding profiles. Similar to a previously described $\mathrm{GH} 26$-associated $\mathrm{CBM} 27^{36}$, RiCBM27 preferred mannohexaose $\left(\mathrm{M}_{6}\right)\left(K_{\mathrm{d}}=165 \pm 10 \mu \mathrm{M}\right.$, two independent experiments, \pm indicates standard deviation), (Table 1, Supplementary Fig. 5a) and its affinity dropped for ligands smaller than a tretrasaccharide (Table 1). By contrast, RiCBM23 was selective for shorter oligosaccharides with its highest affinity for $\mathrm{M}_{4} \quad\left(K_{\mathrm{d}}=130 \pm 50 \mu \mathrm{M}\right.$, two independent experiments) (Table 1, Supplementary Fig. 5b), although mannotriose $\left(M_{3}\right)$ was also bound with good affinity (Table 1).

Internalization of break-down products from $\boldsymbol{\beta}$-mannan. Within the MULL cluster, the three genes (ROSINTL182_05477 ROSINTL182_05479) that encode an ATP-binding cassette
Table 1 Binding parameters of RiCBM27 and RiCBM23 to manno-oligosaccharides and cello-oligosaccharides

\begin{tabular}{|c|c|c|}
\hline \multirow[b]{2}{*}{ Ligand } & \multicolumn{2}{|l|}{$K_{\mathrm{d}}(\mu \mathrm{M})$} \\
\hline & RiCBM27 & RiCBM23 \\
\hline$M_{3}$ & $1593 \pm 30$ & $230 \pm 20$ \\
\hline $\mathrm{M}_{4}$ & $658 \pm 20$ & $130 \pm 50$ \\
\hline $\mathrm{M}_{5}$ & $321 \pm 20$ & $198 \pm 70$ \\
\hline$M_{6}$ & $165 \pm 10$ & $205 \pm 40$ \\
\hline $\mathrm{Glc}_{4}$ & No binding & No binding \\
\hline $\mathrm{Glc}_{6}$ & No binding & No binding \\
\hline \multicolumn{3}{|c|}{$\begin{array}{l}\text { Binding was determined by SPR. Values show the means and standard deviations of at least two } \\
\text { independent experiments } \\
K_{d} \text { dissociation constant }\end{array}$} \\
\hline
\end{tabular}

Table 2 Thermodynamic binding parameters of RiMnBP to linear and decorated manno-oligosaccharides

\begin{tabular}{|c|c|c|c|c|c|}
\hline Ligand & $\begin{array}{l}K_{\mathrm{d}} \\
(\mu \mathrm{M})\end{array}$ & $\begin{array}{l}\Delta G \\
\text { (kcal } \\
\text { mol-1) }^{-1}\end{array}$ & $\begin{array}{l}\Delta H \\
\left(\mathrm{kcal}^{-1}\right. \\
\left.\mathrm{mol}^{-1}\right)\end{array}$ & $\begin{array}{l}-T \Delta S \\
\text { (kcal } \\
\left.\mathrm{mol}^{-1}\right)\end{array}$ & $n$ \\
\hline$M_{3}$ & 2.62 & -7.6 & -33.2 & 25.6 & 0.7 \\
\hline $\mathrm{M}_{4}$ & 3.89 & -7.4 & -28.6 & 21.2 & 0.7 \\
\hline$M_{5}$ & 2.55 & -7.7 & -21.8 & 14.1 & 0.8 \\
\hline$M_{6}$ & 33.75 & -6.2 & -17.8 & 11.6 & 0.5 \\
\hline $\mathrm{M}_{4} \mathrm{Ac}_{2}$ & 25.65 & -6.3 & -21.9 & 15.6 & 0.9 \\
\hline $\mathrm{M}_{5} \mathrm{Ac}_{2}$ & 23.53 & -6.3 & -20.2 & 13.9 & 0.8 \\
\hline
\end{tabular}

Binding was measured by ITC. Data are means of two independent titrations $K_{d}$ dissociation constant, $\Delta G$ Gibbs free energy, $\Delta H$ enthalpy, $-T \Delta S$ entropy, $n$ binding stoichiometry

(ABC) importer were shown to exhibit the highest level of increased expression during growth on $\beta$-mannan (and when compared to growth on glucose). The thermodynamic binding parameters of the ABC-transporter associated solute binding protein, RiMnBP, to linear and substituted mannooligosaccharides were determined using isothermal titration calorimetry (ITC). RiMnBP bound a range of unsubstituted manno-oligosaccharide with a preference for $\mathrm{M}_{5}\left(K_{\mathrm{d}}\right.$ of $\left.2.55 \mu \mathrm{M}\right)$ followed by $\mathrm{M}_{3}$ and $\mathrm{M}_{4}$ (Table 2, Supplementary Fig. 6). Acetylations had a marginal effect on the binding affinities, thus providing evidence that these fragments are efficiently captured by the transport protein. Overall, these results support the predicted role of RiMnBP in the uptake of manno-oligosaccharides generated by RiGH26, showing optimal affinity for undecorated or acetyl substituted ligands with a degree of polymerization (DP) of $4-5$.

Decomposition of internalized $\boldsymbol{\beta}$-manno-oligosaccharides. The affinity of the solute binding protein RiMnBP to mannooligosaccharides and the predicted intracellular location of the debranching and exo-acting enzymes is consistent with a hierarchical degradation of the internalized manno-oligosaccharides following extracellular degradation of the $\beta$-mannan polymers by RiGH26.

The ROSINTL182_05471 (RiCEX) and ROSINTL182_05473 (RiCE2) gene products possess SGNH hydrolase-type esterase domain signatures ${ }^{37}$. Comparison to previously characterized CEs revealed that $\mathrm{RiCE} 2$ showed $25-30 \%$ identity to a CE2 from Clostridium thermocellum ${ }^{38}$ and the acetyl xylan esterase Axe2C of Cellvibrio japonicus ${ }^{38}$. In contrast, RiCEX did not display 
a

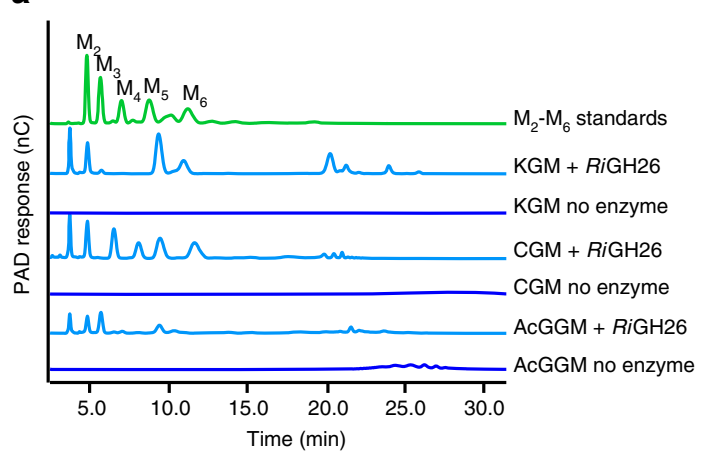

C
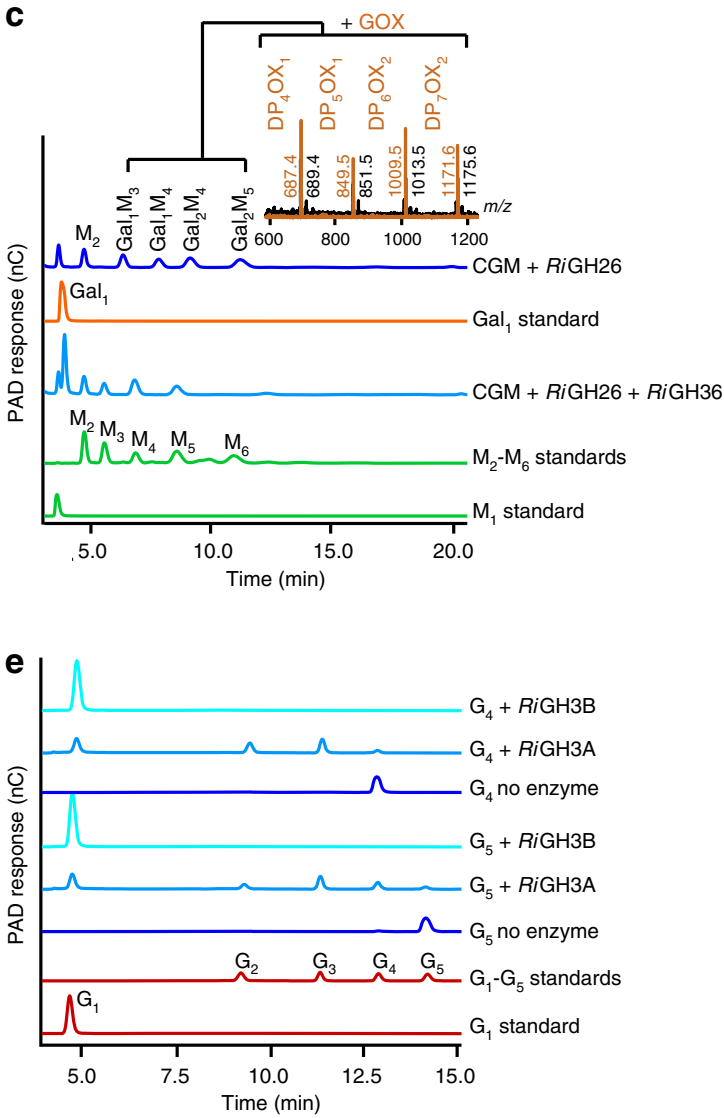

b

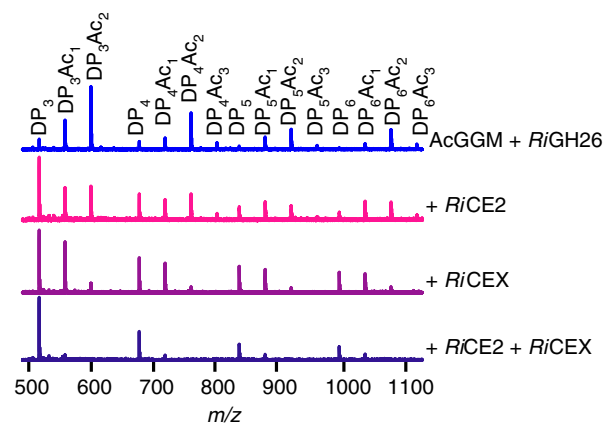

d
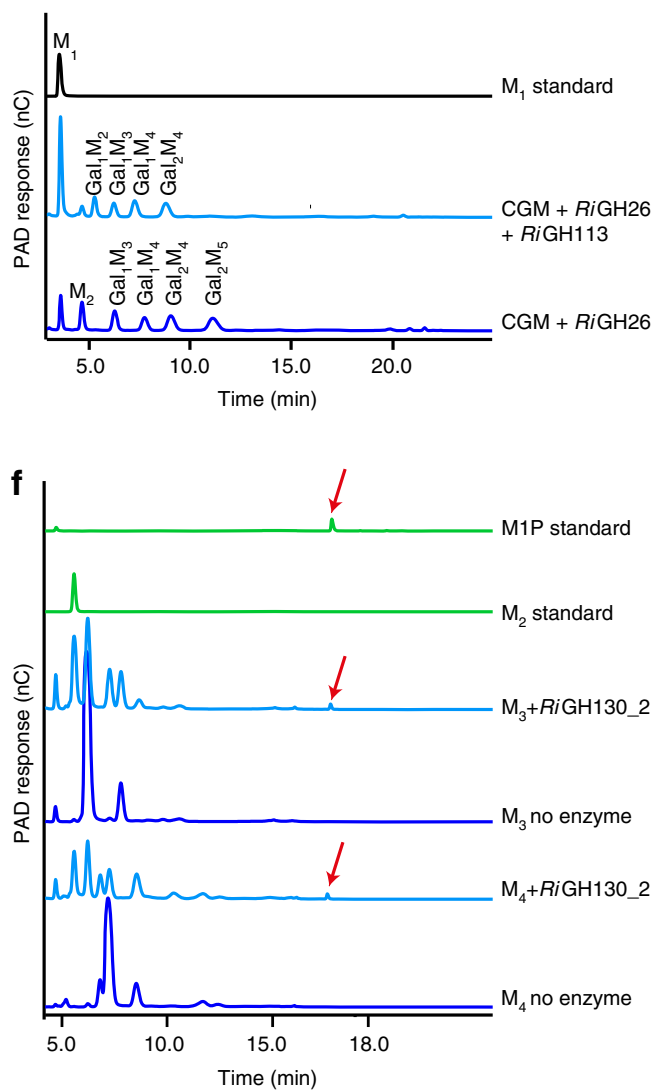

Fig. 4 Cleavage of the $\beta$-mannans backbone, removal of the side chains and further depolymerization of the resulting linear manno-oligosaccharides. a HPAEC chromatograms showing the oligosaccharide products after overnight digestion of KGM, CGM and AcGGM with RiGH26. Samples were analyzed with the following manno-oligosaccharides as external standards: $M_{2}$, mannobiose; $M_{3}$, mannotriose; $M_{4}$, mannotetraose; $M_{5}$, mannopentaose; $M_{6}$, mannohexaose. b MALDI-TOF analysis of RiGH26-digested AcGGM incubated with either RiCE2, RiCEX or both enzymes. Peaks are labeled by DP and number of acetyl groups (Ac). c HPAEC chromatograms showing products generated from CGM pre-digested with RiGH26 and subsequently treated with RiGH36. Assignments for peaks not occurring in the standard samples are based on comparison with the product profiles obtained by MALDI-TOF MS of RiGH26-digested CGM (black spectrum) treated with galactose oxidase (GOX; brown spectrum). GOX converts a galactose residue in the oligosaccharides into its corresponding aldehyde, giving a mass-to-charge ratio $(\mathrm{m} / \mathrm{z})$ of -2 . All assigned masses are sodium adducts. Abbreviations: Ox, oxidation; $\mathrm{Gal}_{1}$, galactose; $\mathrm{Gal}_{1} \mathrm{M}_{3}$, galactosylmannotriose; $\mathrm{Gal}_{1} \mathrm{M}_{4}$, galactosylmannotetraose; $\mathrm{Gal}_{2} \mathrm{M}_{4}$, digalactosylmannotetraose; $\mathrm{Gal}_{2} \mathrm{M}_{5}$, digalactosylmannopentaose. d Product profiles from RiGH26-digested CGM degradation experiments with RiGH113 analyzed by HPAEC-PAD. The release of mannose confirms the exo-activity of RiGH113. e HPAEC-PAD traces showing activity of RiGH3A or RiGH3B towards $\mathrm{G}_{5}$ and $\mathrm{G}_{4}$ with the corresponding controls (no enzyme). Product profiles at various time points during the reaction are shown in Supplementary Fig. 6 . Taken together, the data show that RiGH3B is able to hydrolyze completely both tetramers and pentamers, producing glucose. RiGH3A shows exo-activity towards both substrates that are converted slowly to glucose and a mixture of cello-oligosaccharides. Samples were analyzed with the following cello-oligosaccharides as external standards: glucose, $\mathrm{G}_{1}$; cellobiose, $\mathrm{G}_{2}$; cellotriose, $\mathrm{G}_{3}$; cellotetraose, $\mathrm{G}_{4}$; cellopentaose, $\mathrm{G}_{5}$. f Chromatograms showing products generated upon incubation of RiGH130_2 with $\mathrm{M}_{4}$ and $\mathrm{M}_{3}$. The M1P released (red arrow) was identified by co-migration with the appropriate standard. In all panels, the data displayed are representative of at least three biological triplicates 
significant relatedness to other characterized CE catalogued in the CAZy database ${ }^{33}$, which excluded RiCEX from being classified in any of the 16 CE families. RiCEX and RiCE2 showed mannan acetyl esterase activity on a mixture of oligosaccharides generated via RiGH26 hydrolysis of AcGGM (Fig. 4b). RiCE2 partially removed acetyl groups from the acetylated oligosaccharide substrate (Fig. 4b). RiCEX deacetylated the substrate mainly to free and monoacetylated oligosaccharides (Fig. 4b). These results indicate that RiCEX has a preference for oligosaccharides with a degree of acetyl substitution $\geq 2$, but is less efficient on monosubstituted substrates. At the same time, it suggests that an acetyl group present at a specific position $(O-2$ or $O-3)$ is resistant to enzymatic deacetylation by RiCEX. The combination of RiCEX and RiCE2 resulted in the almost complete deacetylation of the manno-oligosaccharides, indicating a cooperative interaction of the two esterases (Fig. 4b).

RiGH36 released galactose from internally substituted CGM and AcGGM after the treatment with the RiGH26 $\beta$-mannanase (Fig. 4c and Supplementary Fig. 7). Interestingly, RiGH36 released galactose from CGM-endomannanase products with $100 \%$ efficiency (Fig. 4c, Supplementary Fig. 8a) as no oxidized product could be observed after treatment of these samples with galactose oxidase. The enzyme exhibited limited activity on large polymers (Supplementary Fig. 8b) consistent with the activity on internalized oligosaccharides in vivo. Similarly, a-galactosidase activity increased after de-acetylation of the oligosaccharides (Supplementary Fig. 8c, d). Beside cleaving single internal galactose residues from manno-oligosaccharides, this enzyme was capable of removing a-1,6-galactose from the reducing-end of a substituted manno-oligosaccharide (Supplementary Fig. 8e) and from an oligosaccharide containing two consecutive substitutions (Supplementary Fig. 8f). Corroborating these results, RiGH36 cleaved galactose decorations from endomannanase products of highly substituted guar gum galactomannan (Supplementary Fig. 8b).

Sequence searches showed that the protein encoded by ROSINTL182_05483 (MULL, RiGH113) exhibited 40\% identity to the only characterized enzyme from this family, the endo- $\beta$ mannanase AaManA from Alicyclobacillus acidocaldarius ${ }^{39}$ (Supplementary Fig. 9a). Alignment of RiGH113 and AaManA showed that the catalytic and substrate interacting residues are conserved (Supplementary Fig. 9a). When RiGH113 was assayed for activity on linear manno-oligosaccharides, it revealed an ability to cleave linear manno-oligosaccharides to yield mannose and $\mathrm{M}_{2}$ (Supplementary Fig. 9b). Strikingly, time-course analysis of RiGH113 activity revealed that this enzyme displays a different sub-specificity by hydrolyzing manno-oligosaccharides to mannose and a minor amount of $\mathrm{M}_{2}$ (Supplementary Fig. 9c). After overnight incubation with $\mathrm{RiGH} 113, \mathrm{M}_{2}$ was partially degraded to mannose (Supplementary Fig. 9d), confirming the exomannosidase activity as opposed to the endo-fashion cleavage reported for the AaManA. The substituted mannooligosaccharides galactosylmannobiose $\left(\mathrm{Gal}_{1} \mathrm{Man}_{2}\right)$ and digalactosylmannopentaose $\left(\mathrm{Gal}_{2} \mathrm{Man}_{5}\right)$ were hydrolyzed to a lesser extent than non-substituted substrates (Supplementary Fig. 9e); no activity could be detected on $\mathrm{Gal}_{1} \mathrm{Man}_{2}$ while $\mathrm{Gal}_{2} \mathrm{Man}_{5}$ was hydrolyzed to yield mannose and digalactosylmannotetraose $\left(\mathrm{Gal}_{2} \mathrm{Man}_{4}\right)$, which was resistant to further hydrolysis. When the reducing end of manno-oligosaccharides was blocked (Supplementary Fig. 10a-d), no RiGH113 activity could be detected demonstrating that this enzyme possesses a previously unknown reducing end mannose-releasing exo-oligomannosidase activity. Consistent with the view that RiGH113 is an intracellular enzyme, release of mannose was detected after incubation of the enzyme with RiGH26-generated CGM-oligosaccharides (Fig. 4d). The closest homologs of this enzyme are encoded by Clostridium cluster XIVa strains and a range of Firmicutes (Supplementary Fig. 10e).

Product analysis of end point assays and a time course study revealed that both $\mathrm{RiGH} 3 \mathrm{~A}$ (ROSINTL182_07684) and RiGH3B (ROSINTL182_07685) were $\beta$-glucosidases, with redundancy in structure (Supplementary Fig. 11a, b), active on linear cellooligosaccharides (Fig. 4e). RiGH3B completely hydrolyzed cellotetraose $\left(\mathrm{G}_{4}\right)$ and cellopentaose $\left(\mathrm{G}_{5}\right)$ into glucose monomers, whereas $\mathrm{RiGH} 3 \mathrm{~A}$ released glucose and a range of oligosaccharides with lower efficiency compared to that of RiGH3B (Supplementary Fig. 11c, d). Neither of these enzymes were active on mannooligosaccharides (Supplementary Fig. 11e, f). While RiGH3B was able to hydrolyze glucosylmannose $\left(G_{1} M_{1}\right)$ and, partially, mannosylglucose $\left(\mathrm{M}_{1} \mathrm{G}_{1}\right)$ into monomers (Supplementary Fig. 11f), RiGH3A displayed activity only towards $G_{1} M_{1}$. No activity was detected on polymeric KGM (Supplementary Fig. 12a), while glucose was released after incubation of both RiGH3A and RiGH3B with RiGH26-generated KGM-hydrolysate (Supplementary Fig. 12b). Importantly, the latter results demonstrate that $\mathrm{RiGH} 26$ can accept a glucose moiety at the subsite +1 , generating oligosaccharides with a glucose residue at the non-reducing end.

Recombinant RiGH130_2 (MULL, ROSINTL182_05474) phosphorolyzed $\mathrm{M}_{4}$ into $\overline{\mathrm{M}}_{3}, \mathrm{M}_{2}$ and mannose-1-phosphate (M1P) while $M_{3}$ was processed to $M_{2}$ and M1P (Fig. 4f). The enzyme was inactive on cello-oligosaccharides (Supplementary Fig. 13). RiGH130_2 was functional only in the presence of inorganic phosphate, confirming that RiGH130_2 is a mannosylphosphorylase.

Catabolism of mannobiose and mannosylglucose units. The concerted action of the MULL and MULS encoded enzymes described above on the oligosaccharides generated by RiGH26, suggest an intracellular accumulation of $\mathrm{M}_{2}$. Hydrolysis of this product into monosaccharides is accomplished through the action of two enzymes encoded by the co-transcribed genes ROSINTL182_05476 (RiMep) and ROSINTL182_05475 (RiGH130_1).

RiMep was active on $M_{2}$ and cellobiose $\left(G_{2}\right)$, releasing $M_{1} G_{1}$ and $G_{1} M_{1}$, respectively (Fig. 5a). These data show that RiMep is an enzyme active on the reducing end sugar and catalyzes the conversion of disaccharide substrates to the corresponding C2 epimer. This enzyme exhibited epimerization activity not only for the substrate but also for the product as, under high enzyme amount and long reaction time, it was able to convert $M_{1} G_{1}$ and $G_{1} M_{1}$ to $M_{2}$ and $G_{2}$, respectively (Supplementary Fig. 14a). In addition, RiMep exhibited epimerization activity towards oligosaccharides with a DP $>2$ but not on monosaccharides (Supplementary Fig. 14b).

ROSINTL182_05475 encodes a specific mannosylglucose phosphorylase belonging to the GH130 subfamily $1^{40}$. RiGH130_1 was inactive on $\mathrm{G}_{1} \mathrm{M}_{1}$ and oligosaccharides with a $\mathrm{DP} \geq 2$ (Supplementary Fig. 15). RiGH130_1 displayed activity only towards $M_{1} G_{1}$ in the presence of inorganic phosphate, releasing glucose and M1P (Fig. 5b, c).

Catabolism of phosphorolysis products. RiPgm catalyzes the interconversion of M1P and mannose 6-phosphate (M6P) (Fig. 5d). In addition, the enzyme displayed activity also against D-glucose 1-phosphate (G1P) yielding D-glucose 6-phosphate (G6P) (Supplementary Fig. 16a), thus indicating that RiPgm is a phosphomannomutase (PMM)/phosphoglucomutase (PGM) which can use either glucose or mannose as substrate. Consistent with the presence of a predicted magnesium-binding loop 

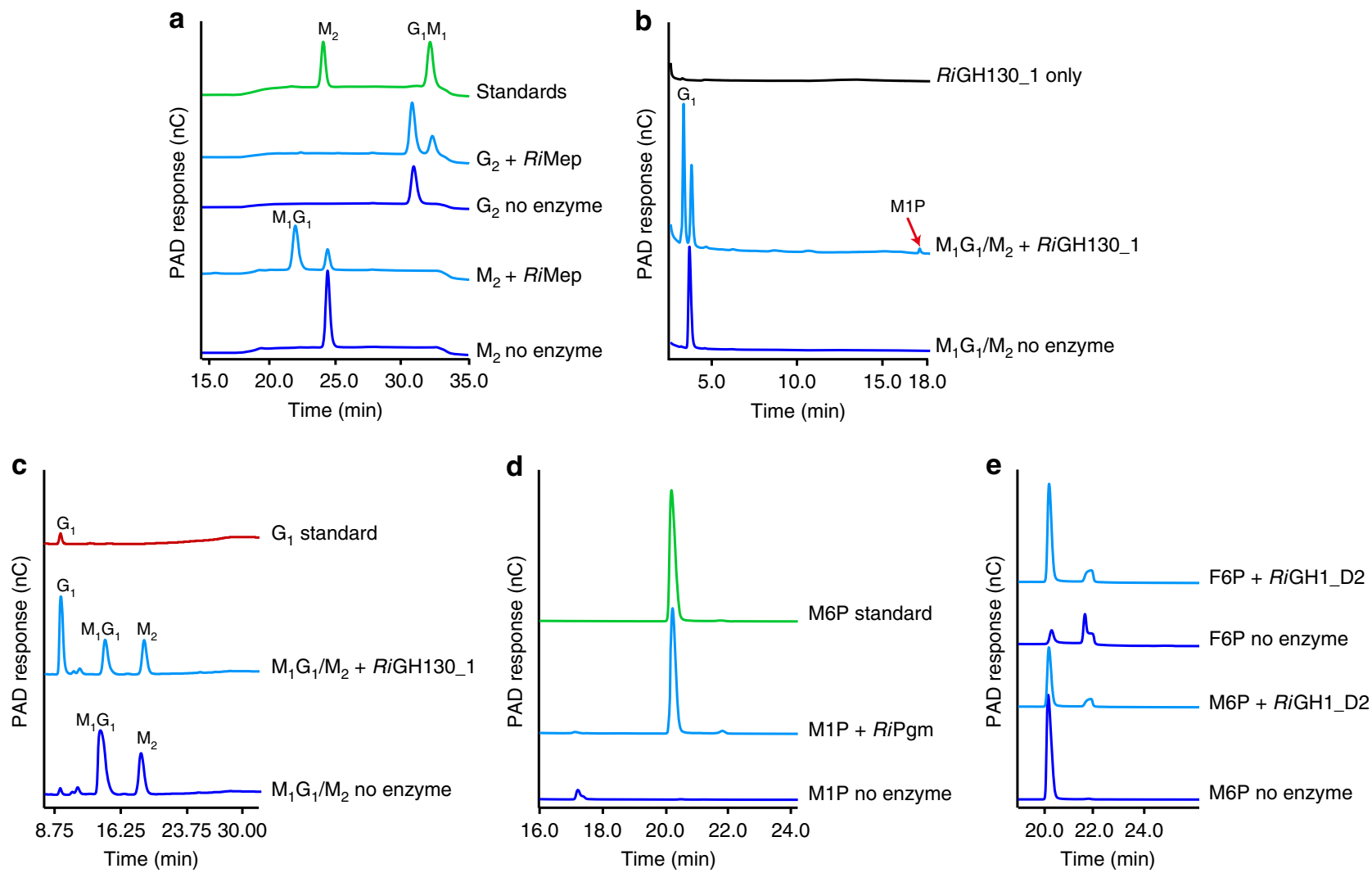

Fig. 5 Enzymes for catabolism of mannobiose, mannosylglucose and monosaccharides deriving from complex $\beta$-mannan degradation. a HPAEC-PAD traces showing the epimerization of $M_{2}$ and $G_{2}$ by RiMep to release $M_{1} G_{1}$ and $G_{1} M_{1}$, respectively. $\mathbf{b}, \mathbf{c}$ HPAEC-PAD of phosphorolysis reactions of RiGH130_1 using premixed $M_{1} G_{1}$ plus $M_{2}$ from Megazyme. The reaction products were then analyzed using an HPAEC method designed for the separation of b phosphorylated manno-oligosaccharides or $\mathbf{c}$ mono-saccharides and di-saccharides. M1P, indicated with a red arrow, and $G_{1}$ peaks in $\mathbf{b}$ are marked according to the standards. d HPAEC-PAD analysis of RiPgm-catalyzed conversion of M1P to M6P. The M6P released was identified by co-migration with the M6P standard. e Activity of RiGH1_D2 on M6P and F6P analyzed by HPAEC-PAD

(Supplementary Fig. 16b), the RiPgm-mediated catalytic activity was detected only when $\mathrm{MgCl}_{2}$ was present in the reaction.

ROSINTL182_05469/70 encodes a predicted bi-functional protein consisting of an $\mathrm{N}$-terminal glucosidase domain (RiGH1_D1, aa 1-246) and a C-terminal family GH1 isomerase domain (RiGH1_D2, aa 247-768). RiGH1_D1 shares $44 \%$ identity to the previously characterized $\beta$-glucosidase TmGH1 from Thermotoga maritima ${ }^{41}$. The recombinant RiGH1_D1 displayed no catalytic activity against all the tested substrates, including $\mathrm{G}_{4}, \mathrm{M}_{4}, \mathrm{M}_{5}, \mathrm{M} 6 \mathrm{P}, \mathrm{G} 6 \mathrm{P}$, and fructose 6-phosphate (F6P). Thus, RiGH1_D1 functional significance is currently unclear. RiGH1_D2 is a phosphomannose isomerase catalyzing the interconversion of M6P into F6P (Fig. 5e).

R. intestinalis competes with Bacteroides for $\beta$-mannans. The ability of $R$. intestinalis to capture, breakdown $\beta$-mannan and efficiently internalize manno-oligosaccharides may increase its fitness to compete with other resident $\beta$-mannan degraders, including the glycan generalist Bacteroides ${ }^{30}$. To test this hypothesis, we performed in vitro co-cultivation of $R$. intestinalis and the efficient $\beta$-mannan degrader Bacteroides ovatus ATCC $8483^{30}$. Both bacteria showed similar growth rates in individual cultures supplemented with AcGGM (Fig. 6a). Population estimates using qPCR indicated that, in the mixed cultures, both $B$. ovatus and $R$. intestinalis grew well during the exponential growth phase, suggesting that the bacteria shared the available carbon source and maintained coexistence. (Fig. 6b). During the stationary phase, when glycan availability is limited, the mean relative abundance of $R$. intestinalis and B. ovatus in the culture was approximately $72.5 \%$ versus $27.5 \%$, respectively. In contrast, $R$. intestinalis showed slow growth on mannose (Fig. 6c) and was outcompeted when co-cultured in this carbon source with $B$. ovatus (Fig. 6d).

$\boldsymbol{R}$. intestinalis responds rapidly to $\boldsymbol{\beta}$-mannan supplementation. To elucidate whether dietary supplementation of $\beta$-mannan can result in expansion of key gut bacteria able to utilize this hemicellulose, germfree mice were colonized with a synthetic microbiota composed of 14 sequenced strains of human commensal gut bacteria $^{42}$. Colonized mice were fed a high-fiber diet for 14 days before being switched to a series of diet regimes with a varying amount of AcGGM (Fig. 6e). Overall, the levels of four species $(R$. intestinalis, Bacteroides uniformis, B. ovatus and Marvinbryantia formatexigens) gradually increased at both AcGGM doses (Fig. 6f-i) and these strains were able to suppress the bacteria foraging on the glyco-protein rich mucus layer (Akkermansia muciniphila, Bacteroides caccae, Bacteroides thetaiotamicron, Bacteroides intestinihominis) (Fig. 6e and j-m) and the amino acids degraders (E. coli, Clostridium symbiosum and Collinsella aerofaciens) (Fig. 6e and $\mathrm{n}$ ). Upon feeding of a fiber-deficient diet, the fecal bacterial abundance of the mucin-eroding bacteria, the sulfate-reducer Desulfovibrio piger and the three amino acid degraders (Fig. 6e) rapidly increased with a corresponding decline of the fiber-degrading species. 

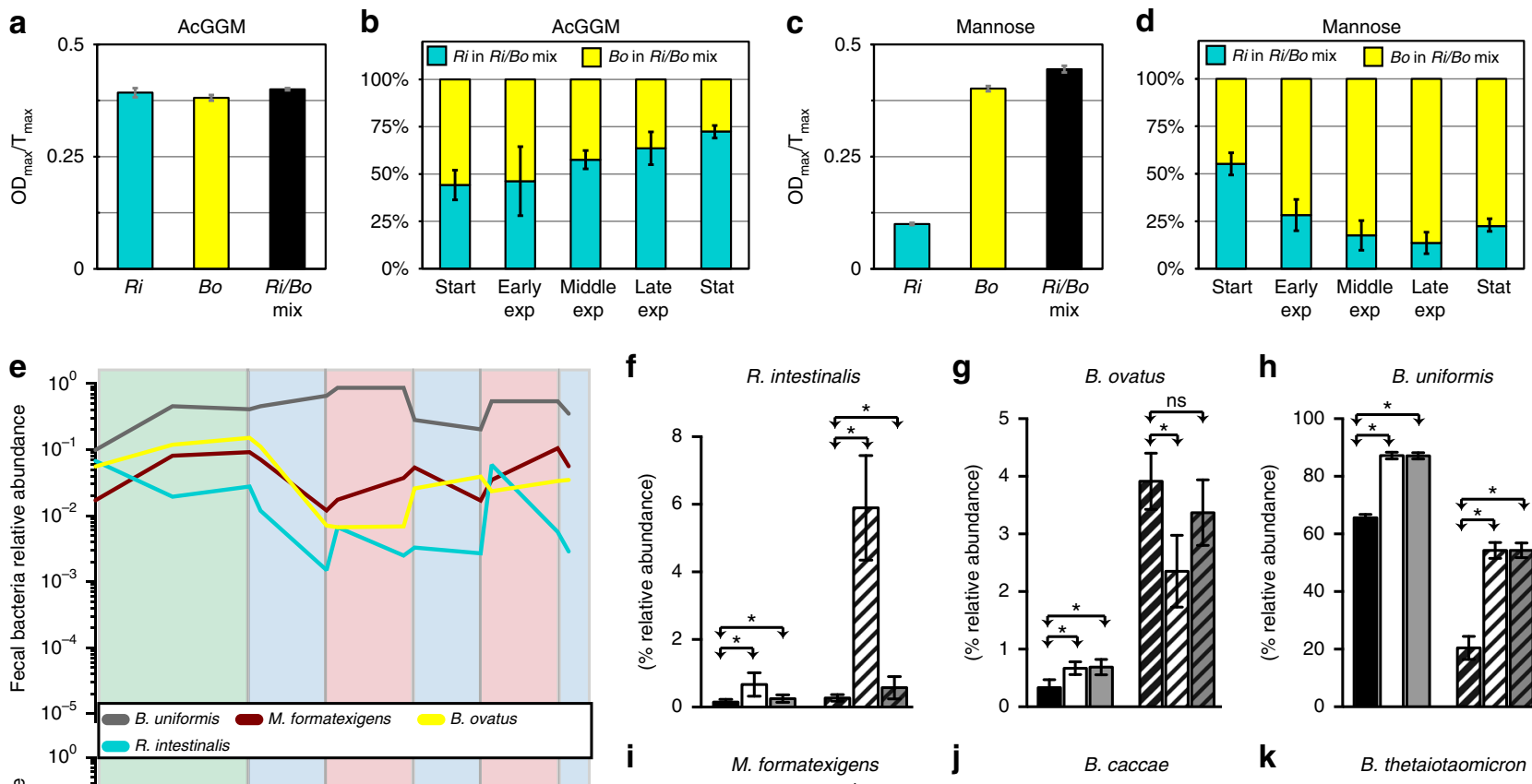

g B. ovatus

h

h $\quad$ B. uniformis
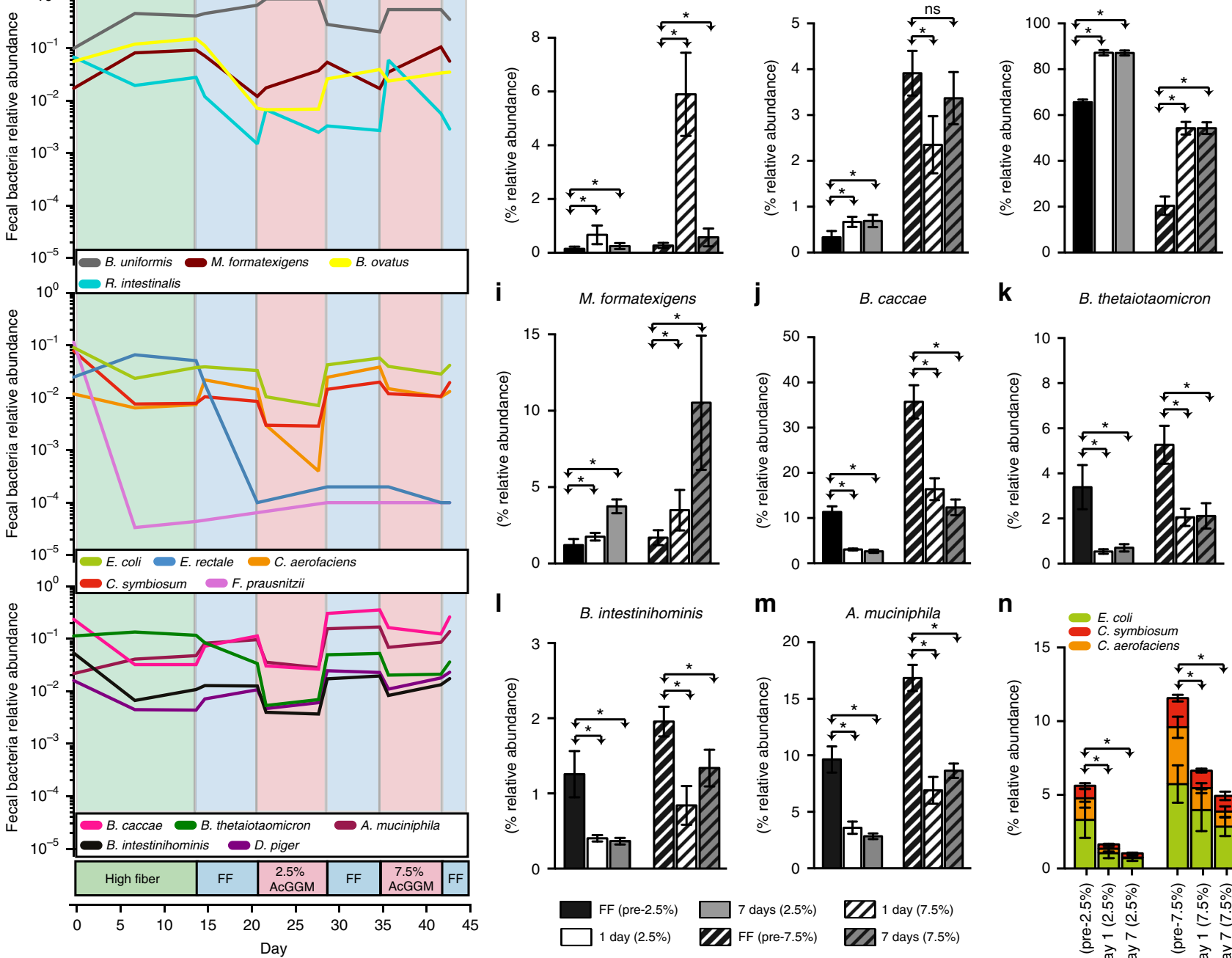

$\mathbf{n}$

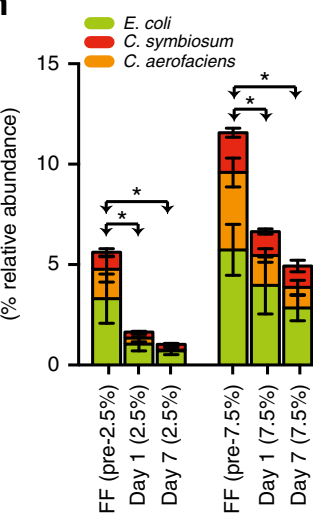

Fig. 6 R. intestinalis and B. ovatus co-culture experiments and in vivo modulation of a synthetic human gut microbiota via AcGGM. a, c Growth rates of mono- and mixed cultures of $R$. intestinalis L1-82 (Ri) and B. ovatus ATCC $8483(\mathrm{Bo})$ on either AcGGM or mannose. Growth rate is defined as the maximum increase in absorbance at $600 \mathrm{~nm}\left(O D_{\max }\right)$ divided by the time ( $T_{\max }$ in hours) to reach the maximum growth. $\mathbf{b}$, $\mathbf{d}$ In vitro competition experiment with $R$. intestinalis L1-82 and B. ovatus ATCC 8483 on either AcGGM or mannose as sole carbon source. The pH of the stationary phase cultures after growth on either AcGGM or mannose was $5.8 \pm 0.16$ and $5.6 \pm 0.11$ (two biological triplicates, \pm indicates the s.d.), respectively, thus showing that the results are not due to differences in acid sensitivity between the two strains. The relative abundance of the bacteria for each different phases of growth was determined by quantitative PCR of species-specific vs universal primers targeting the $16 \mathrm{~S}$ rRNA genes. In a-d, the histogram bars show the mean of two biological replicates, with three independent measurements per replicate. Error bars represent s.d. Abbreviations: Early exp, early exponential phase; Middle Exp; middle exponential phase; Late exp, late exponential phase; Stat, stationary phase. e Relative abundance of bacteria in fecal samples from germfree mice colonized with a synthetic human microbiota. Mice were shifted from a fiber-free (FF) diet to varying amounts ( $2.5 \%$ and $7.5 \% \mathrm{w} / \mathrm{w})$ of AcGGM over time. Data are average of seven mice. $\mathbf{f}-\mathbf{i}$ Relative abundance of individual $\beta$-mannan-degrading bacteria and $\mathbf{j}$-m mucus-degraders. $\mathbf{n}$ Additive relative abundances of three amino acids degraders. In $\mathbf{f}-\mathbf{n}$ histogram bars show the average of seven biological replicates while error bars represent s.d. $P$-values were calculated by two-tailed Student's $t$ test. An asterisk $\left(^{*}\right)$ indicate a statistically significant difference $(P<0.05)$ in the relative abundance of each bacterium compared to that of the specific pre-FF diet. ns, not significant $(P \geq 0.05)$ 


\section{Discussion}

$\beta$-Mannans are widely present in the human diet as constituents of hemicellulose in beans, some nuts and food additives, but are recalcitrant to intestinal digestion by host enzymes. These glycans instead serve as a carbon source for mannanolytic bacteria in the distal gastrointestinal tract, primarily Firmicutes and Bacteroidetes. Recent studies have characterized a few enzymes encoded by two polysaccharide utilization loci (PULs) implicated in the metabolism of galactomannan in B. ovatus ${ }^{30,43}$ and homopolymeric mannan in Bacteroides fragilis ${ }^{32}$. To date, a full understanding of $\beta$-mannan utilization by Firmicutes, however, remains underexplored. The human gut butyrate-producing Firmicute $R$. intestinalis has previously been shown to utilize galactomannan and glucomannan as a carbon source ${ }^{42}$ and possesses predicted genes for the utilization of these substrates ${ }^{24}$. However, no data are available relating the mannanolytic activity at a biochemical level. In this study, we show that two conserved loci, MULL and MULS, collectively provide $R$. intestinalis the capacity to depolymerize this plant polysaccharide. Detailed biochemical studies of the encoded enzymes allowed us to construct a model of sequential action for the mannan utilization system encoded by MULL-MULS (Fig. 7). The RiGH26 and the mannan $\mathrm{ABC}$ uptake system components RiMnBP/RiMPP1/ RiMPP2 transcripts and proteins were the most upregulated in both the RNA sequencing and proteomic analyses, respectively (Fig. 2c, e). This highlights the crucial role of this endomannanase and the $A B C$ transport system in the $\beta$-mannan metabolic pathway. RiGH26 is the only enzyme displayed on the cell surface (Fig. 3), allowing direct access to the intact $\beta$-mannan polymers through dynamic capture mediated by two appended carbohydrate binding modules (RiCBMs). The SPR data showed that RiCBM23 displays $\sim 7$ - and 5-fold higher affinity for $\mathrm{M}_{3}$ and $\mathrm{M}_{4}$, respectively, than RiCBM27, suggesting that the two CBMs play different roles to mediate binding of RiGH26 to mannans. The RiCBMs' $K_{\mathrm{d}}$ values for the preferred manno-oligosaccharides were in the $100-200 \mu \mathrm{M}$ range (Table 1 ). This moderate affinity to the bound substrate constitutes an advantage as it has lower impact on the catalytic activity compared to canonical counterparts from other organisms, and suggests an evolutionary adaptation of $R$. intestinalis to compete with other microbial enzymes

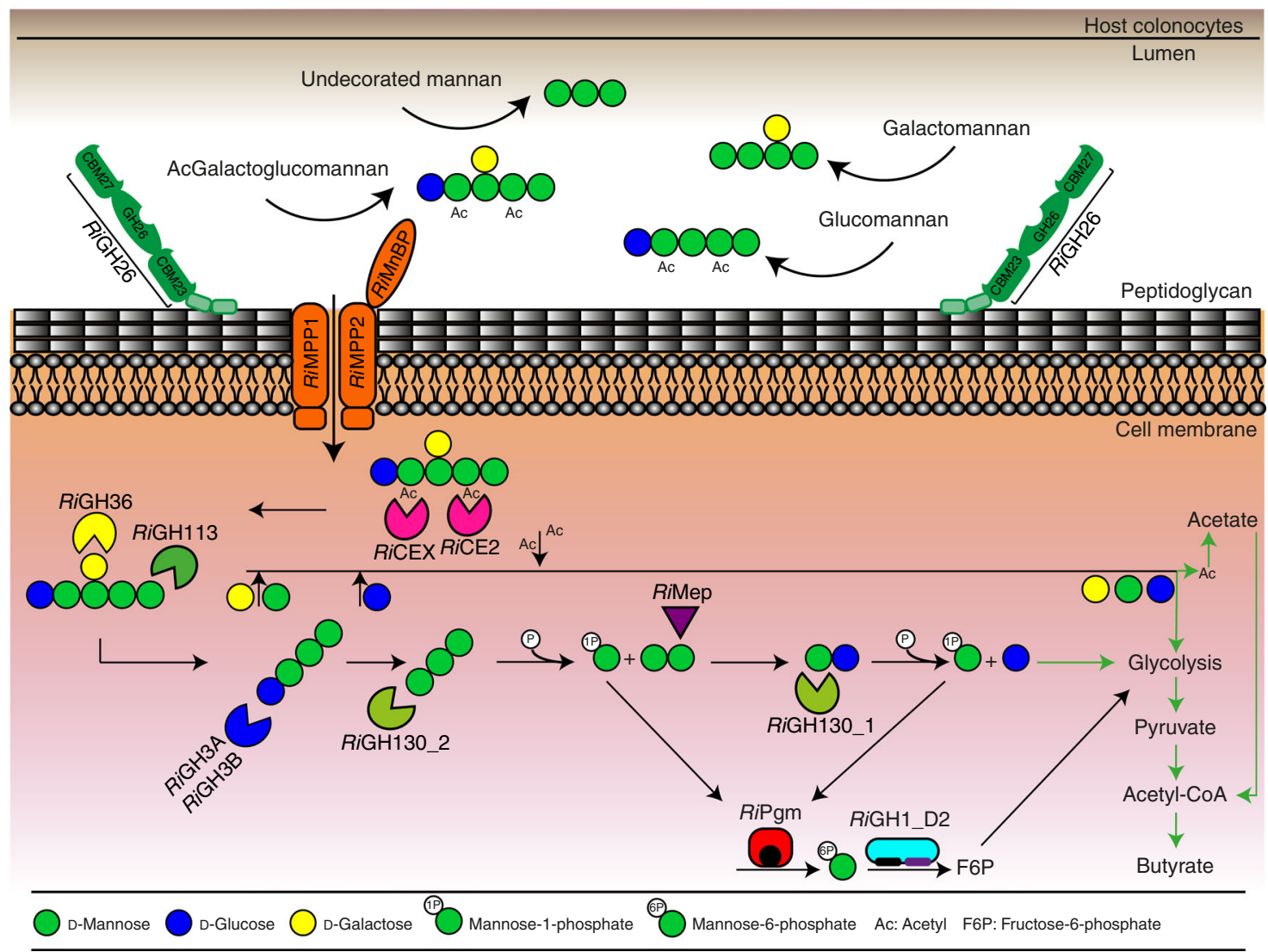

Fig. 7 Model for the degradation and utilization of complex $\beta$-mannans in R. intestinalis. Intracellular degradation of an acetylated galactoglucomannooligosaccharide is used as an example. Sugars are represented as in Fig. 1. Initial depolymerization of acetylated galactoglucomannan (AcGalactoglucomannan) occurs at the outer surface of $R$. intestinalis by the activity of RiGH26 (green). The extracellular recruitment of $\beta$-mannan is facilitated by interactions with CBM27 and CBM23. Import of products occurs through the ABC transporter RiMnBP/RiMPP1/RiMPP2 (orange). Within the cytoplasm, the acetyl and galactosyl decorations are removed by the two acetyl esterases RiCE2 and RiCEX (pink) and the $\alpha$-galactosidase RiGH36 (yellow). The two $\beta$-glucosidases RiGH3A and RiGH3B (blue) release glucose from the non-reducing end of the $\beta$-manno-oligosaccharide. In addition, the reducing end mannose-releasing exo-oligomannosidase RiGH113 (green) can catalyze the removal of mannose units from the decorated mannooligosaccharides until it reaches a galactosyl substituent at the subsite -1 . Once de-ornamented, the $\beta$-manno-oligosaccharides are saccharified by the exo-acting RiGH130_2 (light green) with accumulation of $\mathrm{M}_{2}$. The $\mathrm{M}_{2}$ undergoes subsequent epimerization and phosphorolysis by the concerted activity of RiMep - RiGH130_1 (light green), with release of glucose and M1P. These end products enter the glycolytic pathway either directly (for glucose) or after being converted into M6P and F6P by the phosphomannose mutase RiPgm (red) and the isomerase RiGH1_D2 (turquoise, purple domain). Released mannose is converted to M6P by a hexokinase and processed as described above. Galactose enters glycolysis after conversion to G1P via the Leloir pathway. The pyruvate generated from glycolysis is converted to acetyl-CoA and then butyrate. Black arrows show reactions demonstrated in this study. Green arrows indicate previously demonstrated steps for the generation of butyrate from monosaccharides fermentation ${ }^{68}$ by $R$. intestinalis 
with canonical higher- affinity CBMs, but with reduced catalytic rates $^{44}$. Reliance on multi-modular cell-wall anchored enzymes is a common feature in Firmicutes; ${ }^{45}$ consistently, RiGH26 organization was primarily found in $\beta$-mannanase from other Roseburia species and members of the Clostridium cluster XIVa (Supplementary Fig. 4, Supplementary Table 3-5). Multiplicity of CBMs provides a contrast with the system for mannan metabolism in Bacteroides ovatus ${ }^{30,43}$, where the binding and catalytic activity are distributed between two surface located binding proteins and the single domain mannanase BoMan26B.

Collectively, our results point to a model in which the smaller manno-oligosaccharides generated by $\mathrm{RiGH} 26$ are imported through a dedicated $\beta$-mannan transport system consisting of RiMnBP/RiMPP1/RiMPP2 (Fig. 7). In the cytoplasm, acetylated and galactosylated manno-oligosaccharides are systematically debranched by RiCE2, RiCEX and RiGH36, and subsequently depolymerized. Removal of glucose units from glucomannanoligosaccharides is carried out by RiGH3A and RiGH3B. Based on the highest transcriptional and protein regulation, the main depolymerization strategy for breakdown of unsubstituted manno-oligosaccharides is mediated by the activity of two synergistic mannoside phosphorylases (RiGH130_2 and RiGH130_1) and an epimerase (RiMep), similar to the mannan catabolic pathway proposed in the ruminal bacterium Ruminococcus albus ${ }^{46}$. A similar system has been reported in B. fragilis ${ }^{32}$ and $B$. ovatus $^{30}$, although only composed of an epimerase and a mannosylglucose phosphorylase (GH130_1) that, together, process GH26s-generated $\mathrm{M}_{2}$ units. The presence of the mannooligosaccharide phosphorylases RiGH130_2 allows $R$. intestinalis to process undecorated manno-oligosaccharide of $\mathrm{DP}>2$, consistent with the internalization of large manno-oligosaccharides generated by RiGH26-hydrolysis of polymeric mannan. However, GH130_2s mainly catalyze the phosphorolysis of undecorated manno-oligosaccharides ${ }^{47}$. Removal of mannose units from substituted substrates is mediated by the reducing end mannosereleasing exo-oligomannosidase $\mathrm{RiGH} 113$, which displays a previously undescribed specificity. The two different approaches based on the phosphorylases and the GH113 are likely to be a functional adaptation to accelerate the depolymerization process of mannan. Eventually, mannan catabolism fuels monosaccharide fermentation via glycolysis and leads to the production of butyrate, which is the primary energy source for host colonocytes ${ }^{5,48}$. Colonocytes oxidize butyrate to carbon dioxide ${ }^{49}$, thereby keeping the epithelium hypoxic $\left(<1 \% \mathrm{O}_{2}\right)$. This condition promotes gut homeostasis by stabilizing the hypoxia-inducible transcription HIF that coordinates barrier protection in the mucosa ${ }^{50,51}$. Recently, it has been shown that antibiotic-mediated depletion of butyrate-producing Clostridia increases colonocytes oxygenation and drives aerobic pathogen expansion in the gut lumen, resulting in Salmonella enterica-induced gastroenteritis ${ }^{52}$. Importantly, $R$. intestinalis has been found to affect host histone epigenetic states, direct colonic epithelial cells metabolism away from glycolysis and towards fatty acid metabolism, reduce the levels of inflammatory markers and ameliorate atheriosclerosis in a dietdependent fashion ${ }^{18}$. The athero-protective effect was in part attributed to butyrate, as this SCFA has been shown to inhibit key inflammatory pathways involved in cardiovascular disease development ${ }^{18}$.

The absence of oligosaccharides from $R$. intestinalis AcGGMspent supernatant (Supplementary Fig. 1a, b) demonstrates that the $\beta$-mannan degradation apparatus is optimized for efficient uptake of all the products released by $R i G H 26$, maximize intracellular breakdown and avoid nutrient leakage. This will limit the access to other bacteria, such as Bacteroides spp., competing for the same resource. Using AcGGM, we have shown that $R$. intestinalis and B. ovatus, which possesses an equally complex $\beta$-mannan degrading system, shared the available resources and maintained coexistence (Fig. 6b). Notably, $R$. intestinalis outcompeted $B$. ovatus in the late exponential and stationary phase of growth; these results show that $R$. intestinalis is capable to bind and import the remaining $\beta$-mannan breakdown products (preferred by the RiMnBP transport protein) more efficiently than $B$. ovatus. Thus, it is likely that the $\beta$-mannan utilization apparatus provides $R$. intestinalis with a selective advantage during nutrient limitation when microbial competition for the available carbohydrates in the gut is intense. Understanding the mechanism by which $\beta$-mannan is degraded by key commensal members of the gut is crucial to designing intervention strategies through the use of targeted prebiotics which aim to program or reprogram the composition of the microbiota to maximize human health. Our in vivo study demonstrates that a diet supplemented with AcGGM can be used to manipulate the gut microbiota and to facilitate the growth of species equipped with a $\beta$-mannan degrading system, including $R$. intestinalis (Fig. 6e). This is supported by the increase in the relative abundance of $R$. intestinalis, $B$. uniformis and $B$. ovatus, which all possess enzymes able to degrade AcGGM (BACUNI_00371 - BACUNI_00383; BACOVA_02087-02097 and BACOVA_03386-03406 respectively). $R$. intestinalis was highly responsive to the AcGGM within a day, with a 10 to 30 fold increase at the $2.5 \%$ and $7.5 \%$ AcGGM diet, corroborating its ability to respond dynamically to variation in this dietary fiber. Intriguingly, $R$. intestinalis' response did not last over the 7 day feeding treatment and the acetogen $M$. formatexigens seemed to replace it. A cluster of genes with predicted functions in $\beta$-manno-oligosaccharide utilization (BRYFOR_07194- BRYFOR_07206) was identified in the genome of M. formatexigens (Supplementary Fig.17a). The results shown in Supplementary Fig. 17b,d suggest that $R$. intestinalis and $M$. formatexigens occupy different metabolic niches in the distal gut; the former consumes complex $\beta$-mannans, whereas the acetogen feasts on mono- and oligosaccharides. When in co-culture with either $R$. intestinalis or B. ovatus, $M$. formatexigens was outcompeted in vitro (Supplementary Fig. 17e-f). A previous study with gnotobiotic mice bi-associated with the prominent saccharolytic bacterium $B$. thetaiotamicron and $M$. formatexigens showed that the presence of $M$. formatexigens caused a decrease in the cecal levels of $B$. thetaiotaomicron, compared with monoassociated controls. Transcriptional and metabolic analyses demonstrated that $M$. formatexigens is capable of consuming a variety of plant-derived oligosaccharides and microbial and hostderived $N$-glycans (such as $N$-acetylglucosamine), suggesting that this ability could confer a fitness advantage when competing with the glycan-consuming Bacteroides ${ }^{53}$. Thus, it is likely that, when present as part of the synthetic microbial community described in this paper, $M$. formatexigens may be indirectly benefiting of either manno-oligosaccharides feeding/cross-feeding with other microorganisms or by its ability to grow mixotrophically, simultaneously utilizing organic carbon sources and formate or $\mathrm{H}_{2}$ for energy $^{53}$. Notably, M. formatexigens outcompeted B. ovatus at the 7.5\% AcGGM diet, underscoring the competitiveness of this acetogen in a community setting. In the context of a complex microbial community, it is likely that $M$. formatexigens makes an important contribution to host nutrition improving fermentation by acting as a formate or $\mathrm{H}_{2}$ sink and by generating acetate as main metabolic product ${ }^{53}$.

Collectively, diet-induced changes involved the promotion of mannanolytic bacteria producing propionate, acetate and butyrate, metabolites that are known to regulate hepatic lipid, glucose homeostasis and health of the intestinal hepithelium ${ }^{11}$. These SCFA-producers gained a competitive advantage over colonic mucin-degrading bacteria. Given that intermittent dietary fiberdeprivation results in a thinner mucus layer in mice, eventually 
enhancing pathogen susceptibility ${ }^{42}$, our results support the concept that $\beta$-mannan-based interventions not only could contribute to preventing mucus barrier dysfunctions but also maintaining a gut environment that keeps pathogenic bacteria away. If confirmed in humans, these findings may help to prevent diseases affecting the integrity of the colonic mucus layer, such as ulcerative colitis ${ }^{54}$. Indeed, the fact that the $\beta$-mannan degradation pathway is a core trait found in the majority of the human gut microbiota ${ }^{55}$ highlights the relevance of potential therapeutic interventions through the use of $\beta$-mannan formulations to the general population.

\section{Methods}

Glycans. Carbohydrate substrates used in this study are listed in Supplementary Table 6. All glycan stocks were prepared at $10 \mathrm{mg} \mathrm{ml}^{-1}$ in $\mathrm{ddH}_{2} \mathrm{O}$ and sterilized by filtration using a $0.22 \mu \mathrm{m}$ membrane filter (Sarstedt AG \& Co, Germany).

Bacterial strains and growth conditions. Unless otherwise stated, $R$. intestinalis $\mathrm{L} 1-82^{23}$ was routinely grown at $37^{\circ} \mathrm{C}$ without agitation in an anaerobic cabinet (Whitley A95 workstation, Don Whitley, UK) under an $85 \% \mathrm{~N}_{2} / 10 \% \mathrm{H}_{2} / 5 \% \mathrm{CO}_{2}$ atmosphere. Growth experiments were carried out in YCFA medium (YCFAYeast extract-Casein hydrolysate-Fatty Acids $)^{56}$ supplemented with $0.5 \%(\mathrm{w} / \mathrm{v})$ of the specific carbohydrate to be examined. Overnight cultures ( $300 \mu \mathrm{l})$ were used to inoculate $30 \mathrm{ml}$ aliquots of YCFA plus the carbohydrate to be tested. These precultures were passaged at least three times on the same media to ensure cell growth adaptation on a single carbon source prior to inoculation of the final cultures for growth experiments, RNA-sequencing and proteomic analysis. Bacterial growth was determined spectrophotochemically by monitoring changes in the optical density at $600 \mathrm{~nm}\left(\mathrm{OD}_{600}\right)$. In addition, growth on turbid substrates was assessed by measuring differences in $\mathrm{pH}$ compared to that of starting medium. Growth and $\mathrm{pH}$ curves are averages of three biological replicates, with two technical replicates each. Routine culturing of Bacteroides ovatus ATCC 8483 and M. formatexigens DSM 14469 was in anaerobic Chopped Meat Medium ${ }^{57}$ under static conditions at $37^{\circ} \mathrm{C}$.

Transcriptomic analysis by RNA sequencing. $R$. intestinalis was cultured in triplicate on YCFA supplemented with $0.5 \%(\mathrm{w} / \mathrm{v})$ glucose, galactose, KGM or AcGGM as described above. Cells were harvested at mid-exponential phase and RNA was extracted using the RNeasy Mini Kit (Quiagen) according to the manufacturer's instructions. RNA-seq libraries were prepared using the ScriptSeq Complete kit from Epicentre. Samples were paired-end sequenced on an Illumina Hiseq 4000 instrument at Beijing Genomics Institute (BGI). Analysis of the RNAseq results was performed exactly as described $i^{25}$. Differential gene expression analysis was performed with the DeSeq2 package ${ }^{58}$.

Cloning, overexpression, and protein purification. The genes encoding mature forms of the proteins described in this study were amplified from the $R$. intestinalis L1-82 genomic DNA (BioProject accession number PRJNA30005 [https://www. ncbi.nlm.nih.gov/bioproject/PRJNA30005]) by PCR, using appropriate primers (Supplementary Table 7). PCR products were generated using the Q5 High-Fidelity DNA Polymerase (New England BioLabs, United Kingdom) with 50 ng DNA as template. Prior to cloning the DNA fragment encoding RiGH1_D2 (ROSINTL182 05469), sequence ambiguities at the 3'-end of ROSINTL182 05470 were corrected through sequencing the PCR product generated with the primers listed in the Supplementary Table 8. The gene ROSINTL182_07683 was synthesized without the N-terminal signal sequence predicted by SignalP v.4. ${ }^{59}$ (residues 1-27 from transcription start). The PCR amplicons were cloned into the pNIC-CH expression vector by ligation-independent cloning (LIC) ${ }^{60}$. The gene encoding RiMnbp (ROSINTL182_05479) was cloned in the vector pETM-11 following the method described elsewhere ${ }^{25}$. Recombinant proteins generally contained a Cterminal $\mathrm{His}_{6}$-tag, although, in some cases, His-tag translation was prevented by the introduction of one or two stop codons at the end of the open-reading frame (RiMep, RiGH36, RiPgm and RiGH113). The His 6 -tag was excluded to prevent interaction with putative C-terminal active or catalytic residues that could be detrimental to the enzymes' activity. Constructs were verified by sequencing (Eurofins, UK). Proteins were produced in E. coli BL21 Star (DE3) cells (Invitrogen) as previously described ${ }^{61}$. Briefly, cells were cultured to mid-exponential phase in Tryptone Yeast extract (TYG) containing $50 \mathrm{mg} \mathrm{ml}^{-1}$ kanamycin at $25^{\circ} \mathrm{C}$. Protein overexpression was induced by adding isopropyl $\beta$-D-

thiogalactopyranoside (IPTG) to a final concentration of $200 \mu \mathrm{M}$, followed by incubation for a further $16 \mathrm{~h}$ at $25^{\circ} \mathrm{C}$. Cells were harvested by centrifugation, sonicated and recombinant proteins were purified by either immobilized metal ion affinity chromatography (IMAC) or hydrophobic interaction chromatography (HIC). For IMAC purification, the clarified cell lysate was loaded onto $5 \mathrm{ml}$ HisTrap HP Ni Sepharose columns (GE Healthcare) connected to an ÄKTA purifier FPLC system (GE Healthcare). Protein elution was achieved by using a linear gradient from 5 to $500 \mathrm{mM}$ imidazole. RiGH113, RiGH36, RiMep and RiPgm were purified by HIC by loading the cell-free broth, adjusted to buffer A (1.5 M ammonium sulfate), onto a $5 \mathrm{ml}$ HiTrap Phenyl FF (GE Healthcare) equilibrated with the same buffer. Protein was eluted by using a linear reverse gradient to $100 \mathrm{mM} \mathrm{NaCl}$ over $90 \mathrm{~min}$ at a flow rate of $2.5 \mathrm{ml} \mathrm{min}^{-1}$. After IMAC and HIC, samples were concentrated and further purified by size exclusion chromatography (SEC) using a HiLoad 16/60 Superdex G75 size exclusion column (GE Healthcare) and a running buffer consisting of $20 \mathrm{mM}$ Tris- $\mathrm{HCl} \mathrm{pH} 8.0$ with $200 \mathrm{mM}$ $\mathrm{NaCl}$. Fractions containing the pure protein were combined, concentrated and buffer exchanged to $20 \mathrm{mM}$ Tris pH 8.0, using a Vivaspin 20 (10-kDa molecular weight cutoff) centrifugal concentrators (Sartorius Stedim Biotech GmbH, Germany). Protein purity was estimated to be over $95 \%$ for all the enzymes using SDSPAGE. Protein concentrations were determined using the Bradford assay (Bio-Rad, Germany)

Glycoside hydrolase and phosphorylase activity assays. Enzyme assays, unless otherwise stated, were carried out in $10 \mathrm{mM}$ sodium phosphate buffer, $\mathrm{pH} 5.8$, for up to $16 \mathrm{~h}$ at $37^{\circ} \mathrm{C}$ and $700 \mathrm{rpm}$. Reactions with RiGH130_1 and RiGH130_2 were prepared in $100 \mathrm{mM}$ sodium phosphate buffer, $\mathrm{pH} 5.8$. The activity of RiPgm against M1P and G1P was tested in $10 \mathrm{mM}$ sodium phosphate buffer, $\mathrm{pH}$ 5.8, supplemented with $1 \mathrm{mM} \mathrm{MgCl}$. To determine the specificity of RiGH113, the recombinant protein was sequentially incubated with $0.1 \mathrm{mg} \mathrm{ml}^{-1}$ pre- reduced or oxidized mannooligosaccharides at $37^{\circ} \mathrm{C}$ overnight. Reduction of manno-oligosaccharides was conducted by incubating $1 \mathrm{mg} \mathrm{ml}^{-1}$ manno-oligosaccharides in a volume of $75 \mu \mathrm{l}$ with sodium borodeuteride $\left(\mathrm{NaBD}_{4} ; 0.5 \mathrm{M}\right.$ in $\left.100 \mathrm{mM} \mathrm{NaOH}\right)$ solution. The reaction was incubated overnight at room temperature then quenched by adding $25 \mu \mathrm{l}$ of $25 \mathrm{mM}$ sodium acetate. Oxidation of manno-oligosaccharides reducing-end was obtained by incubating the substrates $\left(1 \mathrm{mg} \mathrm{m}^{-1}\right)$ with the Neurospora crassa cellobiose dehydrogenase $(\mathrm{NcCDH})$ overnight at $37^{\circ} \mathrm{C}$. Both $\mathrm{NaBD}_{4}$ and $\mathrm{NcCDH}$ pretreated samples were diluted 10X in standard assay buffer before addition of RiGH113. Between three and five independent experiments were performed to determine the enzyme activities.

MALDI-TOF mass spectrometry of reaction products. Reaction products generated by the enzymes used in this study were analyzed by matrix-assisted laser desorption/ionization time of flight mass spectrometry (MALDI-TOF MS) as described previously ${ }^{62}$. Briefly, $2 \mu \mathrm{l}$ of a matrix, consisting of $9 \% 2,5$-dihydroxybenzoic acid (DHB) in 30\% acetonitrile, were applied to an MTP 384 ground steel target plate TF (Bruker Daltonics, Germany). Sample (1 $\mu \mathrm{l})$ was then mixed with the matrix and dried under a stream of warm air. Samples were analyzed with an Ultraflex MALDI-ToF/ToF instrument (Bruker Daltonics, Germany), equipped with a Nitrogen $337 \mathrm{~nm}$ laser beam and operated in positive acquisition mode. Results were analyzed using the Bruker FlexAnalysis software (version 3.3).

HPAEC-PAD. Mono- and oligosaccharides products were analyzed on a Dionex ICS-3000 HPAEC system operated by the Chromeleon software version 7 (Dionex Thermo Scientific), as described previously ${ }^{62}$. Sugars were injected onto a CarboPac PA1 $2 \times 250$-mm analytical column (Dionex, Thermo Scientific) coupled to a CarboPac PA1 $2 \times 50-\mathrm{mm}$ guard column kept at $30^{\circ} \mathrm{C}$. Manno-oligosaccharides and phosphorylated monosaccharides were eluted in $0.1 \mathrm{M} \mathrm{NaOH}$ at a flow rate of $0.25 \mathrm{ml} \mathrm{min}^{-1}$ by increasing the concentration of sodium acetate ( $\mathrm{NaOAc}$ ) exponentially from 0 to $0.3 \mathrm{M}$ over $26 \mathrm{~min}$ (from 9 to $35 \mathrm{~min}$ after injection), before column reconditioning by $0.1 \mathrm{M} \mathrm{NaOH}$ for $10 \mathrm{~min}$. Commercial mannooligosaccharides with DP2 -6 were used as standards. For cello-oligosaccharides, the separation was done using a multistep linear gradient going from $0.1 \mathrm{M} \mathrm{NaOH}$ to $0.1 \mathrm{M} \mathrm{NaOH}-0.1 \mathrm{M} \mathrm{NaOAc}$ over $10 \min , 0.1 \mathrm{M} \mathrm{NaOH}-0.14 \mathrm{M} \mathrm{NaOAc}$ after $14 \mathrm{~min}, 0.1 \mathrm{M} \mathrm{NaOH}-0.3 \mathrm{M} \mathrm{NaOAc}$ at $16 \mathrm{~min}$ followed by a 2 min exponential gradient to $1 \mathrm{M} \mathrm{NaOAc}$, before reconditioning with $0.1 \mathrm{M} \mathrm{NaOH}$ for 9 min. Cello-oligosaccharides with DP 2-6 were used as standards. For the analysis of disaccharides $\left(G_{1} M_{1}\right.$ or $\left.M_{1} G_{1}\right)$ and phosphorylated monosaccharides generated from the activity of RiGH130_2, RiMep, RiGH130_1, RiPgm and RiGH1, the elution was done at $0.25 \mathrm{ml} \mathrm{min}^{-1}$ using a $40 \mathrm{~min}$ program. The program started with $0.01 \mathrm{M}$ potassium hydroxide $(\mathrm{KOH})$ for $15 \mathrm{~min}$, reaching the concentration of $0.1 \mathrm{M} \mathrm{KOH}$ at $25 \mathrm{~min}$ after injection and was kept for additional $5 \mathrm{~min}$ at the same $\mathrm{KOH}$ concentration. Between each sample, the column was re-equilibrated by running initial conditions for $10 \mathrm{~min}$.

Protein cellular localization. Proteins of interest were detected using anti-sera raised in rats (Eurogentec) against the corresponding recombinant RiGH26 or the previously characterized RiXyn $10 \mathrm{~A}^{25}$

For immunofluorescence microscopy, $R$. intestinalis cells were grown in YCFA containing $0.5 \%$ AcGGM, wheat arabinoxylan (WAX) or glucose to an $\mathrm{OD}_{600}$ of 0.8 , collected by centrifugation $(4000 \times g$ for $5 \mathrm{~min}$ ) and washed twice in phosphate buffered saline (PBS). Cells were resuspended in $500 \mu \mathrm{l}$ PBS and fixed by adding an equal volume of $2 \times$ formalin ( $9 \%$ formaldehyde in PBS) on ice for $30 \mathrm{~min}$. The bacterial pellet was washed twice with $1 \mathrm{ml}$ PBS prior to resuspension in $1 \mathrm{ml}$ of blocking buffer ( $1 \%$ bovine serum albumin, BSA, in PBS) and incubation at $4{ }^{\circ} \mathrm{C}$ for $16 \mathrm{~h}$. After incubation the cell pellets were harvested by centrifugation and the supernatant discarded. For labelling, the bacteria were incubated with $0.5 \mathrm{ml}$ of anti-sera (diluted 1:500 in blocking buffer) for $2 \mathrm{~h}$ at $25^{\circ} \mathrm{C}$. The cells were then 
pelleted, washed with $1 \mathrm{ml}$ PBS and resuspended in $0.5 \mathrm{ml}$ goat anti-rat IgG AlexaFluor 488 (Sigma-Aldrich), diluted 1:500 in blocking buffer and incubated $1 \mathrm{~h}$ at $25^{\circ} \mathrm{C}$. The cells were again harvested, washed with $1 \mathrm{ml} \mathrm{PBS}$ and suspended in 100 $\mu \mathrm{l}$ PBS containing one drop of ProLong Gold antifade reagent (Life Technologies). Labeled bacterial cells were mounted onto glass slides and secured with coverslips. Fluorescence microscopy was performed on a Zeiss AxioObserver equipped with the ZEN Blue software. Images were acquired using an ORCA-Flash4.0 V2 Digital CMOS camera (Hamamatsu Photonics) through a 100x phase-contrast objective. A HXP 120 Illuminator (Zeiss) was used as a fluorescence light source.

\section{Analysis of the bacterial proteome. $R$. intestinalis was grown in triplicate on} YCFA supplemented with either $0.5 \%(\mathrm{w} / \mathrm{v})$ glucose or AcGGM, respectively, as a sole carbon source. Samples $(10 \mathrm{ml})$ were harvested at the mid-exponential growth phase. Cell pellet was collected by centrifugation $\left(4500 \times g, 10 \mathrm{~min}, 4^{\circ} \mathrm{C}\right)$, resuspended in $50 \mathrm{mM}$ Tris- $\mathrm{HCl}, 0.1 \%(\mathrm{v} / \mathrm{v})$ Triton X-100, $200 \mathrm{mM} \mathrm{NaCl}, 1 \mathrm{mM}$ dithiothreitol and disrupted by bead-beating using three $60 \mathrm{~s}$ cycles with a FastPrep24 (MP Biomedicals, CA). Proteins were precipitated with ice-cold trichloroacetic acid (TCA), final concentration of $10 \%(\mathrm{v} / \mathrm{v})$, incubated on ice for $1 \mathrm{~h}$, centrifuged $\left(15,000 \times g, 15 \mathrm{~min}, 4^{\circ} \mathrm{C}\right)$ to pellet the precipitated proteins and washed with $300 \mu \mathrm{l}$ ice-cold $0.01 \mathrm{M} \mathrm{HCl}$ in $90 \%$ acetone. Proteins were separated by SDSPAGE with a $10 \%$ Mini-PROTEAN gel (Bio-Rad Laboratories, CA) and then stained with Coomassie brilliant blue R250. The gel was cut into five slices, after which proteins were reduced, alkylated, and in-gel digested according to a method published previously ${ }^{63}$. The peptides were dried under vacuum, solubilized in $0.1 \%$ (v/v) trifluoroacetic acid (TFA) and desalted using $\mathrm{C}_{18}$ ZipTips (Merck Millipore, Germany) according to the manufacturer's instructions.

The peptide mixture from each fraction was analyzed using a nanoHPLC-MS/ MS system as described previously ${ }^{63}$, using a Q-Exactive hybrid quadrupoleorbitrap mass spectrometer (Thermo Scientific) equipped with a nano-electrospray ion source. Mass spectral data were acquired using Xcalibur (v.2.2 SP1).

MS raw files were processed with the MaxQuant software suite ${ }^{64}$ (version 1.4.1.2) for identification and label-free quantification (LFQ) of proteins. Proteins were identified by searching MS and MS/MS data of peptides against the UniProtKB complete proteome of $R$. intestinalis L1-82 (4698 sequences) supplemented with common contaminants (e.g., keratins, trypsin, and bovine serum albumin). In addition, reversed sequences of all protein entries were concatenated to the database for estimation of false-discovery rates (FDRs). Trypsin was set as proteolytic enzyme and two missed cleavages were allowed. Protein N-terminal acetylation, oxidation of methionines, deamidation of asparagines and glutamines and formation of pyro-glutamic acid at $\mathrm{N}$-terminal glutamines were defined as variable modifications while carbamidomethylation of cysteines was used as a fixed modification. The "match between runs" feature of MaxQuant, which enables identification transfer only between samples from the same carbon source based on accurate mass and retention time, was applied with default parameters. All identifications were filtered in order to achieve a protein FDR of $1 \%$. A protein was considered "present" if it was detected in at least two of the three biological replicates in at least one glycan substrate. Missing values were imputed from a normal distribution (width of 0.3 and down shifted 1.8 standard deviations from the original distribution) in total matrix mode and differential abundance analysis was performed using an unpaired two-tailed Student's $t$-test with a permutation-based FDR set to 0.05 . Hierarchical clustering and heat map representations were generated using Euclidean distance measure and average linkage using Perseus ${ }^{65}$ (version 1.5.5.3).

Substrate binding assay using SPR. The affinity of $R i \mathrm{CBM} 27$ and $R i \mathrm{CBM} 23$ to soluble manno-oligosaccharides and cello-oligosaccharides was evaluated by SPR using a Biacore T100 (GE Healthcare). The two CBMs, diluted into $10 \mathrm{mM}$ sodium acetate ( $\mathrm{pH} 4.1$ ) to $2.3 \mu \mathrm{M}$, were immobilized on a NTA sensor chip (GE Healthcare) to a density of 3000-4000 response units (RU). Sensograms were recorded at $25^{\circ} \mathrm{C}$ in phosphate/citrate buffer $(20 \mathrm{mM}$ phosphate/citrate buffer; $150 \mathrm{mM} \mathrm{NaCl} ; \mathrm{pH} 6.5,0.005 \%$ (v/v) P20 surfactant) at $30 \mu \mathrm{l}$ per min with association and dissociation times of $90 \mathrm{~s}$ and $240 \mathrm{~s}$, respectively. CBMs binding was tested towards $0.2 \mathrm{nM}-1 \mathrm{mM}$ of carbohydrate ligands dissolved in the same buffer as above. Data were analyzed using the Biacore T100 evaluation software, and equilibrium dissociation constants $\left(K_{\mathrm{d}}\right)$ were obtained by fitting a single-site binding model to either the steady-state response data or the full sensograms.

ITC. Binding of manno-oligosaccharides to RiMnBP was measured at $25^{\circ} \mathrm{C}$ in $10 \mathrm{mM}$ sodium phosphate $\mathrm{pH} 6.5$ using an $\mathrm{ITC}_{200}$ microcalorimeter (MicroCal). $R i \mathrm{MnBP}$ in the sample cell was titrated by 19 injections of carbohydrate ligand separated by $120 \mathrm{~s}$. The following concentrations were used: $900 \mu \mathrm{M}$ of $\mathrm{M}_{3}$ in the syringe and $76.5 \mu \mathrm{M}$ RiMnBP in the sample cell; $1365 \mu \mathrm{M}$ of $\mathrm{M}_{4}$ or $\mathrm{M}_{5}$ in the syringe and $91 \mu \mathrm{M}$ RiMnBP in the sample cell; $2270 \mu \mathrm{M}$ of $\mathrm{M}_{6}$ in the syringe and $117 \mu \mathrm{M} R i \mathrm{MnBP}$ in the sample cell; $750 \mu \mathrm{M}$ of diacetylated mannotetraose $\left(\mathrm{M}_{4} \mathrm{Ac}_{2}\right)$ in the syringe and $50 \mu \mathrm{M} R i \mathrm{MnBP}$ in the cell; $1500 \mu \mathrm{M}$ of diacetylated mannopentaose $\left(\mathrm{M}_{5} \mathrm{Ac}_{2}\right)$ in the syringe and $100 \mu \mathrm{M}$ RiMnBP in the cell. Thermodynamic binding parameters were determined using the MicroCal Origin software (version $7.0)$
Competition experiments. $R$. intestinalis, B. ovatus and $M$. formatexigens cells were grown overnight under anaerobic conditions in YCFA containing $0.5 \%(\mathrm{w} / \mathrm{v})$ AcGGM (YCFA-AcGGM) as the sole carbon source. These subcultures were used to inoculate, in approximately equal proportions (estimated by $\mathrm{OD}_{600}$ ), $30 \mathrm{ml}$ of the same medium. A control culture of YCFA-AcGGM was also inoculated with either $R$. intestinalis, B. ovatus or M. formatexigens. Growth $\left(\mathrm{OD}_{600}\right)$ was monitored for up to $24 \mathrm{~h}$, withdrawing $1 \mathrm{ml}$ samples for quantitative PCR (qPCR) analysis at selected time points. Cells were pelleted, combined with $200 \mu \mathrm{l}$ of TE buffer ( $\mathrm{pH}$ 7.8) and bead-beated for $2 \mathrm{~min}$ (FastPrep96, MP Biomedicals, CA) using $\leq 106 \mu \mathrm{m}$ acid-washed glass beads (Sigma-Aldrich). Genomic DNA was extracted using the Mag Midi kit (LGC Group, UK) according to the manufacturer's instructions. qPCR was performed in a LightCycler 480 II system (Roche, Germany) using specific primers for each strain (Supplementary Table 9). In addition, a highresolution melting (HRM) analysis was conducted to evaluate the specificity of the amplification and the lack of primer dimers. The raw data were imported into the LinReg PCR program ${ }^{66}$ and the calculated Cq values and PCR efficiency were used to deduce the ratio of R. intestinalis, B. ovatus and M. formatexigens at each time point. Statistically significant differences were determined using the unpaired twotailed Student's $t$-test.

Human gut microbiota-associated mice and diets. All experiments involving animals complied with all relevant ethical regulations for animal testing and research and were approved by the University of Michigan, University Committee for the Use and Care of Animals. Germfree mice were colonized with a synthetic microbiota composed of 14 fully sequenced human species according to the methodology previously adopted by Desai et $\mathrm{a}^{42}$. Briefly, seven 6 -week-old germfree male wild-type Swiss Webster mice that had been raised on ad libitum access to a high fiber chow diet (LabDiet 5013) and autoclaved distilled water were gavaged for 3 consecutive days with $200 \mu \mathrm{l}$ each day of a mixture of the 14 different species. Colonized mice were maintained on this high fiber diet for 14 days before being switched to a series of diet regimes with varying fibers. This feeding sequence consisted of 7 days of feeding on a gamma-irradiated fiber-free (FF) diet (TD.140343, Harlan Teklad, USA) that does not contain AcGGM or related molecules. Mice were then switched for 7 days to a custom version of the same diet that contained AcGGM at $2.5 \% \mathrm{w} / \mathrm{w}$, followed by a 7 -day washout period on the FF diet, and finally 7 days of feeding on a version of this diet containing AcGGM at $7.5 \% \mathrm{w} / \mathrm{w}$ (in both AcGGM diets an equivalent amount of glucose was removed to accommodate the prebiotic addition). Fecal samples were taken 1 day before and 1 day after each diet transition, effectively allowing us to measure changes in response to AcGGM supplementation at 1 and 7 days post exposure relative to the FF diet. The relative abundance of each microbial strain at sampled time points was measured by qPCR, using previously validated primer sets, from total DNA extracted from freshly voided fecal pellets (stored at $-20^{\circ} \mathrm{C}$ until extraction) exactly as described previously ${ }^{42}$. Statistically significant differences were determined using the unpaired two-tailed Student's $t$-test.

Comparative genomic analysis. Identification of similar $\beta$-mannan catabolic genes in bacteria belonging to the Clostridium XIVa cluster was performed using the Gene Ortholog Neighborhood viewer on the Integrated Microbial Genomes website (https://img.jgi.doe.gov). This was done using the genes encoding RiGH26 (ROSINTL182_07683, GenBank ABYJ02000124.1:7167-11129) and RiMEP (ROSINTL182_05476, GenBank ABYJ02000025.1:3200-4429) as the search homolog and the default threshold e-value of 1e-5. Then, a sequence comparison was conducted where each $R$. intestinalis L1-82 RefSeq annotated protein sequence was subjected to BLASTp searches against other Clostridium XIVa members. Sequences with coverage $<60 \%$ and amino acid similarity $<45 \%$ were excluded.

Reporting summary. Further information on experimental design is available in the Nature Research Reporting Summary linked to this article.

\section{Data availability}

All data supporting the findings of this study are available within the article and Supplementary Information, or from the corresponding author upon request. The transcriptomic data described in this article are submitted under NCBI BioProject accession number PRJNA516396. The mass spectrometry proteomics data have been deposited to the ProteomeXchange Consortium via the PRIDE partner repository with the dataset identifier PXD012448.

Received: 18 July 2018 Accepted: 3 February 2019

Published online: 22 February 2019

\section{References}

1. Backhed, F., Ley, R. E., Sonnenburg, J. L., Peterson, D. A. \& Gordon, J. I. Hostbacterial mutualism in the human intestine. Science 307, 1915-1920 (2005). 
2. Flint, H. J., Bayer, E. A., Rincon, M. T., Lamed, R. \& White, B. A. Polysaccharide utilization by gut bacteria: potential for new insights from genomic analysis. Nat. Rev. Microbiol. 6, 121-131 (2008).

3. El Kaoutari, A., Armougom, F., Gordon, J. I., Raoult, D. \& Henrissat, B. The abundance and variety of carbohydrate-active enzymes in the human gut microbiota. Nat. Rev. Microbiol. 11, 497-504 (2013).

4. Hamer, H. M. et al. Review article: the role of butyrate on colonic function. Aliment. Pharmacol. Ther. 27, 104-119 (2008).

5. Roediger, W. E. Role of anaerobic bacteria in the metabolic welfare of the colonic mucosa in man. Gut 21, 793-798 (1980).

6. Wang, H. B., Wang, P. Y., Wang, X., Wan, Y. L. \& Liu, Y. C. Butyrate enhances intestinal epithelial barrier function via up-regulation of tight junction protein Claudin-1 transcription. Dig. Dis. Sci. 57, 3126-3135 (2012).

7. Fung, K. Y., Cosgrove, L., Lockett, T., Head, R. \& Topping, D. L. A review of the potential mechanisms for the lowering of colorectal oncogenesis by butyrate. Br. J. Nutr. 108, 820-831 (2012).

8. Canfora, E. E., Jocken, J. W. \& Blaak, E. E. Short-chain fatty acids in control of body weight and insulin sensitivity. Nat. Rev. Endocrinol. 11, 577-591 (2015).

9. Gibson, G. R. et al. Expert consensus document: The International Scientific Association for Probiotics and Prebiotics (ISAPP) consensus statement on the definition and scope of prebiotics. Nat. Rev. Gastroenterol. Hepatol. 14, 491-502 (2017).

10. Duncan, S. H. et al. Proposal of Roseburia faecis sp. nov., Roseburia hominis sp. nov. and Roseburia inulinivorans sp. nov., based on isolates from human faeces. Int. J. Syst. Evol. Microbiol. 56, 2437-2441 (2006).

11. Louis, P. \& Flint, H. J. Formation of propionate and butyrate by the human colonic microbiota. Environ. Microbiol. 19, 29-41 (2017).

12. Takahashi, K. et al. Reduced abundance of butyrate-producing bacteria species in the fecal microbial community in Crohn's disease. Digestion 93, 59-65 (2016).

13. Chassard, C. et al. Functional dysbiosis within the gut microbiota of patients with constipated-irritable bowel syndrome. Aliment. Pharmacol. Ther. 35 828-838 (2012).

14. Kumari, R., Ahuja, V. \& Paul, J. Fluctuations in butyrate-producing bacteria in ulcerative colitis patients of North India. World J. Gastroenterol. 19, 3404-3414 (2013).

15. Wang, T. et al. Structural segregation of gut microbiota between colorectal cancer patients and healthy volunteers. Isme. J. 6, 320-329 (2012).

16. Geng, J., Fan, H., Tang, X., Zhai, H. \& Zhang, Z. Diversified pattern of the human colorectal cancer microbiome. Gut Pathog. 5, 2 (2013).

17. Atarashi, K. et al. Induction of colonic regulatory $\mathrm{T}$ cells by indigenous Clostridium species. Science 331, 337-341 (2011).

18. Kasahara, K. et al. Interactions between Roseburia intestinalis and diet modulate atherogenesis in a murine model. Nat. Microbiol. 3, 1461-1471 (2018).

19. Jost, T., Lacroix, C., Braegger, C. \& Chassard, C. Assessment of bacterial diversity in breast milk using culture-dependent and culture-independent approaches. Br. J. Nutr. 110, 1253-1262 (2013).

20. Van den Abbeele, P. et al. Arabinoxylans and inulin differentially modulate the mucosal and luminal gut microbiota and mucin-degradation in humanized rats. Environ. Microbiol. 13, 2667-2680 (2011).

21. Van den Abbeele, P. et al. Butyrate-producing Clostridium cluster XIVa species specifically colonize mucins in an in vitro gut model. Isme. J. 7, 949-961 (2013).

22. Nishino, K. et al. Analysis of endoscopic brush samples identified mucosaassociated dysbiosis in inflammatory bowel disease. J. Gastroenterol. 53, 95-106 (2018)

23. Duncan, S. H., Hold, G. L., Barcenilla, A., Stewart, C. S. \& Flint, H. J Roseburia intestinalis sp. nov., a novel saccharolytic, butyrate-producing bacterium from human faeces. Int. J. Syst. Evol. Microbiol. 52, 1615-1620 (2002).

24. Sheridan, P. O. et al. Polysaccharide utilization loci and nutritional specialization in a dominant group of butyrate-producing human colonic Firmicutes. Microb. Genom. 2, e000043 (2016).

25. Leth, M. L. et al. Differential bacterial capture and transport preferences facilitate co-growth on dietary xylan in the human gut. Nat. Microbiol. 3 570-580 (2018).

26. Chassard, C., Goumy, V., Leclerc, M., Del'homme, C. \& Bernalier-Donadille, A. Characterization of the xylan-degrading microbial community from human faeces. FEMS Microbiol. Ecol. 61, 121-131 (2007).

27. Yamabhai, M., Sak-Ubol, S., Srila, W. \& Haltrich, D. Mannan biotechnology: from biofuels to health. Crit. Rev. Biotechnol. 36, 32-42 (2016).

28. Schroder, R., Atkinson, R. G. \& Redgwell, R. J. Re-interpreting the role of endo-beta-mannanases as mannan endotransglycosylase/hydrolases in the plant cell wall. Ann. Bot. 104, 197-204 (2009).

29. Moreira, L. R. \& Filho, E. X. An overview of mannan structure and mannandegrading enzyme systems. Appl. Microbiol. Biotechnol. 79, 165-178 (2008).
30. Bagenholm, V. et al. Galactomannan catabolism conferred by a polysaccharide utilization locus of Bacteroides ovatus: enzyme synergy and crystal structure of a beta-mannanase. J. Biol. Chem. 292, 229-243 (2017).

31. McNulty, N. P. et al. Effects of diet on resource utilization by a model human gut microbiota containing Bacteroides cellulosilyticus $\mathrm{WH} 2$, a symbiont with an extensive glycobiome. PLoS Biol. https://doi.org/10.1371/journal. pbio.1001637 (2013).

32. Kawaguchi, K. et al. The mannobiose-forming exo-mannanase involved in a new mannan catabolic pathway in Bacteroides fragilis. Arch. Microbiol. 196 17-23 (2014)

33. Lombard, V., Golaconda Ramulu, H., Drula, E., Coutinho, P. M. \& Henrissat, B. The carbohydrate-active enzymes database (CAZy) in 2013. Nucleic Acids Res. 42, D490-D495 (2014).

34. Zhou, F., Chen, H. \& Xu, Y. GASdb: a large-scale and comparative exploration database of glycosyl hydrolysis systems. BMC Microbiol. 10, 69 (2010).

35. Navarre, W. W. \& Schneewind, O. Surface proteins of gram-positive bacteria and mechanisms of their targeting to the cell wall envelope. Microbiol. Mol. Biol. Rev. 63, 174-229 (1999).

36. Roske, Y., Sunna, A., Pfeil, W. \& Heinemann, U. High-resolution crystal structures of Caldicellulosiruptor strain Rt8B.4 carbohydrate-binding module CBM27-1 and its complex with mannohexaose. J. Mol. Biol. 340, 543-554 (2004).

37. Akoh, C. C., Lee, G. C., Liaw, Y. C., Huang, T. H. \& Shaw, J. F. GDSL family of serine esterases/lipases. Prog. Lipid Res. 43, 534-552 (2004).

38. Montanier, C. et al. The active site of a carbohydrate esterase displays divergent catalytic and noncatalytic binding functions. PLoS Biol. https://doi. org/10.1371/journal.pbio.1000071 (2009).

39. Zhang, Y. et al. Biochemical and structural characterization of the intracellular mannanase AaManA of Alicyclobacillus acidocaldarius reveals a novel glycoside hydrolase family belonging to clan GH-A. J. Biol. Chem. 283, 31551-31558 (2008)

40. Saburi, W. Functions, structures, and applications of cellobiose 2-epimerase and glycoside hydrolase family 130 mannoside phosphorylases. Biosci. Biotechnol. Biochem. 80, 1294-1305 (2016).

41. Zechel, D. L. et al. Iminosugar glycosidase inhibitors: structural and thermodynamic dissection of the binding of isofagomine and 1deoxynojirimycin to beta-glucosidases. J. Am. Chem. Soc. 125, 14313-14323 (2003).

42. Desai, M. S. et al. A dietary fiber-deprived gut microbiota degrades the colonic mucus barrier and enhances pathogen susceptibility. Cell 167, 1339-1353 (2016).

43. Reddy, S. K. et al. A beta-mannan utilization locus in Bacteroides ovatus involves a GH36 alpha-galactosidase active on galactomannans. FEBS Lett. 590, 2106-2118 (2016).

44. Santos, C. R. et al. Dissecting structure-function-stability relationships of a thermostable GH5-CBM3 cellulase from Bacillus subtilis 168. Biochem. J. 441, 95-104 (2012).

45. Cockburn, D. W. \& Koropatkin, N. M. Polysaccharide degradation by the intestinal microbiota and its influence on human health and disease. J. Mol. Biol. 428, 3230-3252 (2016)

46. Kawahara, R. et al. Metabolic mechanism of mannan in a ruminal bacterium, Ruminococcus albus, involving two mannoside phosphorylases and cellobiose 2-epimerase: discovery of a new carbohydrate phosphorylase, beta-1,4mannooligosaccharide phosphorylase. J. Biol. Chem. 287, 42389-42399 (2012).

47. Ladeveze, S. et al. Role of glycoside phosphorylases in mannose foraging by human gut bacteria. J. Biol. Chem. 288, 32370-32383 (2013).

48. Bar, F. et al. Mitochondrial gene polymorphisms that protect mice from colitis. Gastroenterology 145, 1055-1063 (2013)

49. Donohoe, D. R. et al. The Warburg effect dictates the mechanism of butyrate mediated histone acetylation and cell proliferation. Mol. Cell 48, 612-626 (2012).

50. Kelly, C. J. et al. Crosstalk between microbiota-derived short-chain fatty acids and intestinal epithelial HIF augments tissue barrier function. Cell. Host. Microbe 17, 662-671 (2015).

51. Rivera-Chavez, F., Lopez, C. A. \& Baumler, A. J. Oxygen as a driver of gut dysbiosis. Free Radic. Biol. Med. 105, 93-101 (2017).

52. Rivera-Chavez, F. et al. Depletion of butyrate-producing Clostridia from the gut microbiota drives an aerobic luminal expansion of Salmonella. Cell. Host. Microbe 19, 443-454 (2016).

53. Rey, F. E. et al. Dissecting the in vivo metabolic potential of two human gut acetogens. J. Biol. Chem. 285, 22082-22090 (2010).

54. Johansson, M. E., Sjovall, H. \& Hansson, G. C. The gastrointestinal mucus system in health and disease. Nat. Rev. Gastroenterol. Hepatol. 10, 352-361 (2013).

55. Lloyd-Price, J. et al. Strains, functions and dynamics in the expanded Human Microbiome Project. Nature 550, 61-66 (2017). 
56. Lopez-Siles, M. et al. Cultured representatives of two major phylogroups of human colonic Faecalibacterium prausnitzii can utilize pectin, uronic acids, and host-derived substrates for growth. Appl. Environ. Microbiol. 78, 420-428 (2012).

57. Hehemann, J. H., Kelly, A. G., Pudlo, N. A., Martens, E. C. \& Boraston, A. B. Bacteria of the human gut microbiome catabolize red seaweed glycans with carbohydrate-active enzyme updates from extrinsic microbes. Proc. Natl Acad. Sci. USA 109, 19786-19791 (2012).

58. Love, M. I., Huber, W. \& Anders, S. Moderated estimation of fold change and dispersion for RNA-seq data with DESeq2. Genome Biol. 15, 550 (2014).

59. Petersen, T. N., Brunak, S., von Heijne, G. \& Nielsen, H. SignalP 4.0: discriminating signal peptides from transmembrane regions. Nat. Methods 8, 785-786 (2011).

60. Aslanidis, C. \& de Jong, P. J. Ligation-independent cloning of PCR products (LIC-PCR). Nucleic Acids Res. 18, 6069-6074 (1990).

61. Mackenzie, A. K. et al. A polysaccharide utilization locus from an uncultured bacteroidetes phylotype suggests ecological adaptation and substrate versatility. Appl. Environ. Microbiol. 81, 187-195 (2015).

62. Agger, J. W. et al. Discovery of LPMO activity on hemicelluloses shows the importance of oxidative processes in plant cell wall degradation. Proc. Natl Acad. Sci. USA 111, 6287-6292 (2014)

63. Arntzen, M. O., Karlskas, I. L., Skaugen, M., Eijsink, V. G. \& Mathiesen, G. Proteomic investigation of the response of Enterococcus faecalis V583 when cultivated in urine. PLoS ONE https://doi.org/10.1371/journal.pone.0126694 (2015).

64. Cox, J. \& Mann, M. MaxQuant enables high peptide identification rates, individualized p.p.b.-range mass accuracies and proteome-wide protein quantification. Nat. Biotechnol. 26, 1367-1372 (2008).

65. Tyanova, S. et al. The Perseus computational platform for comprehensive analysis of (prote)omics data. Nat. Methods 13, 731-740 (2016).

66. Ramakers, C., Ruijter, J. M., Deprez, R. H. \& Moorman, A. F. Assumption-free analysis of quantitative real-time polymerase chain reaction (PCR) data. Neurosci. Lett. 339, 62-66 (2003).

67. Varki, A. et al. Symbol nomenclature for graphical representations of glycans. Glycobiology 25, 1323-1324 (2015).

68. Louis, P. \& Flint, H. J. Diversity, metabolism and microbial ecology of butyrate-producing bacteria from the human large intestine. FEMS Microbiol. Lett. 294, 1-8 (2009).

\section{Acknowledgements}

The authors wish to thank M. Kjos (NMBU) for assistance with immunofluorescence microscopy. This work was supported by a grant from the Research Council of Norway (244259). Additional funding was from a Graduate School DTU Scholarship, Lyngby, Denmark for M.L.L. and from the Independent Research Fund Denmark, Natural Sciences (DFF, FNU) by a Research Project 2 grant (Grant ID: 4002-00297B) to M.A.H. Carlsberg Foundation is also acknowledged for an instrument grant for purchase of the ITC200 calorimeter to M.A.H.

\section{Author contributions}

Experiments were primarily designed by S.L.L.R., E.C.M., M.A.H., and B.W. S.L.L.R. cloned, expressed, purified and performed functional characterizations of the enzymes. Production of AcGGM was performed by L.M. and B.W. The initial growth experiments on mannans were performed by M.L.L. and these experiments were used to prepare RNA and performed the transcriptional analysis together with C.T.W. Proteomic analysis was done by S.L.L.R. and M.Ø.A. SPR and ITC was performed by M.E.H., who also cloned, expressed and purified the transport protein. S.L.L.R. and G.P. conducted the in vitro growth experiments, competition experiments and qPCR. S.L.L.R. performed enzyme localization studies. Mice experiment was conducted by N.A.P., R.G. and E.C.M. The manuscript was written primarily by S.L.L.R. and B.W. with contributions from P.B.P., M.Ø.A., M.A.H., and E.C.M. Figures were prepared by S.L.L.R., M.E.H., and N.A.P. All authors reviewed the final manuscript.

\section{Additional information}

Supplementary Information accompanies this paper at https://doi.org/10.1038/s41467019-08812-y.

Competing interests: The authors declare no competing interests.

Reprints and permission information is available online at http://npg.nature.com/ reprintsandpermissions/

Journal peer review information: Nature Communications thanks the anonymous reviewers for their contribution to the peer review of this work. Peer reviewer reports are available.

Publisher's note: Springer Nature remains neutral with regard to jurisdictional claims in published maps and institutional affiliations.

(cc) (i) Open Access This article is licensed under a Creative Commons Attribution 4.0 International License, which permits use, sharing, adaptation, distribution and reproduction in any medium or format, as long as you give appropriate credit to the original author(s) and the source, provide a link to the Creative Commons license, and indicate if changes were made. The images or other third party material in this article are included in the article's Creative Commons license, unless indicated otherwise in a credit line to the material. If material is not included in the article's Creative Commons license and your intended use is not permitted by statutory regulation or exceeds the permitted use, you will need to obtain permission directly from the copyright holder. To view a copy of this license, visit http://creativecommons.org/ licenses/by/4.0/.

(C) The Author(s) 2019 